\title{
SPACES OF COMPLEX NULL GEODESICS IN COMPLEX-RIEMANNIAN GEOMETRY
}

BY

CLAUDE LEBRUN

\begin{abstract}
The notion of a complex-Riemannian n-manifold. meaning a complex n-manifold with a nondegenerate complex quadratic form on each tangent space which varies holomorphically from point to point, is briefly developed. It is shown that, provided $n \geqslant 4$, every such manifold locally arises canonically as the moduli space of all quadrics of a fixed normal-bundle type in an associated space of complex null geodesics. This relationship between local geometry and global complex analysis is stable under deformations.
\end{abstract}

Introduction. The present work provides a general framework analogous to (but distinct from) Penrose's twistor correspondence (Penrose [15], Atiyah et al. [2]) for the study of real-analytic pseudo-Riemannian geometry on manifolds whose dimension exceeds three, in which points of the original manifold correspond to compact complex submanifolds (quadrics) in an associated complex manifold. The natural intermediate step in the construction is to consider complexification of the geometry, leading to the notion of a complex-Riemannian manifold (implicitly present, for instance, in the work of Penrose). In fact, it is really the conformal structure that is of fundamental importance, and so, having briefly introduced holomorphic metrics in Chapter I, we proceed to establish the fundamental facts of holomorphic conformal geometry in Chapter II. Finally, in Chapter III we develop our generalized "twistor" correspondence.

A brief historical note is in order. A special case of this correspondence (where the complex-Riemannian manifold is complex Euclidean 4-space) proved to be useful for the study of Yang-Mills fields (Issenberg et at. [7], Witten [18]) several years ago. In the wake of this discovery, the present author first sketched his ideas on the subject in 1979 in the informal Twistor Newsletter of the Oxford Mathematical Institute; a more fully developed version became the author's Ph. D. thesis (LeBrun [10]), which forms the basis of much of the present, more refined, paper. (Several results are completely new, however, such as the theorems in III.4, 5.) In dimension four, some recent results have shown these notions to be of genuine physical interest; within the last year, a beguiling theory of the Dirac (Manin [13]) and wave (LeBrun [11]) operators, fitting neatly within this general framework, has emerged. For the present, however, we will examine only the big picture, relegating this (arguably more beautiful) restricted case to treatment elsewhere.

Received by the editors March 10, 1982.

1980 Mathematics Subject Classification. Primary 32G10, 53A30; Secondary 32D15, 53C15, 53C22, $83 \mathrm{C} 99$. 
I would like to take this opportunity to express by deepest gratitude to R. Penrose and $\mathrm{N}$. Hitchin for their formative influences, guidance, criticism, and support during my graduate days. I also have greatly appreciated the written comments of Yu. I. Manin and the observations of M. G. Eastwood in many useful discussions. Finally, I would like to extend my most heartfelt thanks to N. H. Kuiper and the Institut des Hautes Études Scientifiques for providing financial support and an ideal environment during the writing of this paper.

\section{COMPLEX-RiemanNian GEOMETRY}

1. Holomorphic metrics. Let $\mathfrak{M}$ be a complex manifold. A holomorphic metric $\mathfrak{g}$ on $\mathfrak{M}$ will mean a holomorphic covariant symmetric 2-tensor $\mathfrak{g} \in \Gamma_{\mathfrak{M}} \odot\left(\odot^{2} T^{* *} \mathfrak{M}\right)$ which

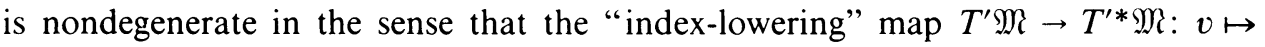
$\mathfrak{g}(v, \cdot)$ is an isomorphism of the holomorphic tangent bundle of $\mathfrak{M}$ to its dual.

If $U \subset \mathfrak{M}$ is a region of $\mathfrak{M}$ parameterized by local holomorphic coordinates $z^{1}, \ldots, z^{n}$, we have

$$
\left.\mathfrak{g}\right|_{U}=\sum_{j, k=1}^{n} \mathfrak{g}_{j k} d z^{j} \otimes d z^{k},
$$

where $\bar{\partial} \mathfrak{g}_{j k}=0, \mathfrak{g}_{j k}=\mathfrak{g}_{k j} \forall j, k$, and $\operatorname{det}\left[\mathfrak{g}_{j k}\right] \neq 0$.

EXAMPLES. The paradigmatic holomorphic metric is that of complex Eudlidean $n$-space $\left(\mathbf{C}^{n}, \sum_{j=1}^{n}\left(d z^{j}\right)^{\otimes 2}\right)$. More generally, let $\mathfrak{M} \subset \mathbf{C}^{n}$ be a complex submanifold, and let $\mathfrak{M}_{0} \subset \mathfrak{M}$ be the open set where the restriction of the complex Euclidean metric $g$ to $\mathfrak{M}$ is nondegenerate; then $\left.g\right|_{\mathfrak{M}_{0}}$ is clearly a holomorphic metric.

Let us emphasize immediately that $g$ is not a pseudo-Riemannian metric on the underlying smooth manifold of $\mathfrak{M}$. Nonetheless, holomorphic metrics are directly relevant to Riemannian geometry. If $(\mathbf{M}, \mathbf{g})$ is a real-analytic pseudo-Riemannian $n$-manifold, we can always find a "complexification" $\alpha: \mathbf{M} \hookrightarrow_{\mathfrak{U}} \mathfrak{M}$, where $\mathfrak{M}$ is a complex $n$-manifold, and then construct a holomorphic metric on some neighborhood of $\mathbf{M}$ such that $\alpha^{*} \mathfrak{g}=\mathbf{g}$; the pair $(\mathfrak{M}, \mathfrak{g})$ is then germ-unique at $\mathbf{M}$. Of course, not all holomorphic metrics arise in this way, since the complexification of a real-analytic pseudo-Riemannian manifold possesses the extra structure (in a suitably "symmetric" neighborhood) of an antiholomorphic involution ("complex conjugation") $\mathfrak{s}: \mathfrak{M} \underset{\overrightarrow{\mathfrak{c}}}{\rightarrow} \mathfrak{M}$ such that $\mathfrak{F}^{*} \mathfrak{g}=\overline{\mathfrak{g}}$ and $\mathfrak{f}^{2}=1$. If $(\mathfrak{M}, \mathfrak{g})$ admits such an involution $\sqrt{ }$, the fixed point set $\mathbf{M}$ will be called the real slice of ( $\mathfrak{M}, \mathfrak{g}$, $\mathfrak{E}$ ) provided that $\mathbf{M} \neq \varnothing$. Such a slice is always naturally a real-analytic pseudo-Riemannian manifold.

We will refer to a pair $(\mathfrak{M}, \mathfrak{g})$, where $\mathfrak{M}$ is a complex manifold and $\mathfrak{g}$ is a holomorphic metric on $\mathfrak{M}$, as a complex-Riemannian manifold.

Notice that a complex manifold $\mathfrak{M}$ will not generally admit any holomorphic metric: for a complex-Riemannian manifold the tangent and cotangent bundles are isomorphic, and hence the odd Chern classes must all be of order 2. (See, e.g., Milnor and Stasheff [14].)

2. Holomorphic affine connections. Let $\mathfrak{M}$ be a complex manifold and $\nabla$ an affine connection on the smooth manifold underlying $\mathfrak{M}$ (i.e. on $\mathfrak{M}$ with its complex 
structure "forgotten"). Extend $\nabla$ to a connection on the complexified tangent bundle $\mathbf{C} T \mathfrak{M}:=\mathbf{C} \otimes_{\mathbf{R}} T \mathfrak{M}$ by linearity and consider the holomorphic tangent bundle $T^{\prime} \mathfrak{M}$ as a vector-subbundle of $\mathbf{C} T \mathfrak{M}$ (namely, as the $(+i)$-eigenspace of the complex structure tensor). We will then say that $\nabla$ is a holomorphic affine connection on $\mathfrak{M}$ iff the covariant derivative of every holomorphic vector-field is a holomorphic tensor-field. A holomorphic affine connection thus defines a holomorphic derivation $\Theta \rightarrow \Omega^{1} \otimes_{\Theta} \Theta$ on the sheaf $\Theta$ of holomorphic vector-fields; conversely, every such derivation arises from a unique holomorphic affine connection. In terms of local coordinates, a holomorphic affine connection appears as

$$
\begin{aligned}
\nabla\left(U^{j} \frac{\partial}{\partial z^{j}}\right. & \left.+U^{j} \frac{\partial}{\partial \bar{z}^{j}}\right)=\left(\frac{\partial U^{j}}{\partial z^{k}} d z^{k}+\frac{\partial U^{j}}{\partial \bar{z}^{k}} d \bar{z}^{-}\right) \otimes \frac{\partial}{\partial z^{j}} \\
+ & \left(\frac{\partial U^{j}}{\partial z^{k}} d z^{k}+\frac{\partial U^{j}}{\partial \bar{z}^{-}} d \bar{z}^{-\bar{k}}\right) \otimes \frac{\partial}{\partial \bar{z}^{j}}+\Gamma_{k l}^{j} U^{l} d z^{k} \otimes \frac{\partial}{\partial z^{j}}+\Gamma_{\bar{k} l}^{j} U^{i} d \bar{z}^{\bar{k}} \otimes \frac{\partial}{\partial \bar{z}^{j}},
\end{aligned}
$$

where the summation convention applies for both barred and unbarred indices, and where

$$
\Gamma_{\frac{j}{k l}}^{\bar{j}}=\overline{\Gamma_{k l}^{j}}, \quad \bar{\partial} \Gamma_{k l}^{j}=0 \quad \forall j, k, l .
$$

By the torsion tensor $T_{\nabla}$ of a holomorphic connection $\nabla$, we shall mean the holomorphic tensor such that $T_{\nabla}+\bar{T}_{\nabla}$ is the torsion of $\nabla$ as a smooth affine connection (see, e.g., Hicks [6]) and hope this will not cause any serious confusion for the reader. (The reason for our convention will become apparent presently.) Thus, our torsion is just the ordinary torsion restricted to the holomorphic tangent bundle, with components given by $T_{k l}^{j}=\Gamma_{k l}^{j}-\Gamma_{l k}^{j}$. Following common usage, a holomorphic connection $\nabla$ will be called symmetric iff $T_{\nabla} \equiv 0$.

Proposition. Let $(\mathfrak{M}, \mathfrak{g})$ be a complex-Riemannian manifold. There is a unique symmetric holomorphic affine connection $\nabla$ on $\mathfrak{M}$ satisfying $\nabla \mathrm{g}=0$.

Proof. Formally identical to the usual theorem of smooth Riemannian geometry (e.g. cf. Hicks [6]). In a coordinate chart the connection symbols $\Gamma_{k l}^{j}$ are given by the Levi-Civita expression

$$
\Gamma_{k l}^{j}=\frac{1}{2} \mathfrak{g}^{j m}\left(\partial_{k} \mathfrak{g}_{l m}+\partial_{l} \mathfrak{g}_{k m}-\partial_{m} \mathfrak{g}_{k l}\right)
$$

where $\partial_{k}:=\partial / \partial z^{k}$ and $\left[\mathfrak{g}^{j k}\right]=\left[\mathfrak{g}_{j k}\right]^{-1}$ as matrices.

We now define the complex geodesics of a complex manifold $\mathfrak{M}$ with holomorphic connection $\nabla$ to be the connected inextendible immersed complex curves which can be given holomorphic parameters $\zeta \in \mathbf{U} \subset \mathbf{C}$ such that $d / d \zeta\lrcorner \nabla(d / d \zeta)=0$, the above expression being independent of the extension of $d / d \zeta$ to the germ of a vector-field on $\mathfrak{M}$. Of course, the underlying real connection of $\nabla$ also has geodesics, which are precisely the real-analytic curves produced by restricting a parameter $\zeta$ of the above type to the real line $\alpha \zeta+\overline{\alpha \zeta}=c \in \mathbf{R}$ for $\alpha \in \mathbf{C} \backslash\{0\}$ a constant; thus, if $J: T \mathfrak{M} \rightarrow T \mathfrak{M}$ is the almost-complex structure-tensor of $\mathfrak{M}$, and if $\exp _{\nabla}: \mathfrak{D} \rightarrow \mathfrak{M}$ is the exponential map of the smooth connection underlying $\nabla$ (where $\mathfrak{D} \subset T \mathfrak{M}$ is the maximal domain of definition), then the unique complex 
geodesic $\gamma$ tangent to $v-i J v, v \in T \mathfrak{M} \backslash \mathbf{0}_{\mathfrak{M}}$ (where $\mathbf{0}_{\mathfrak{M}}$ is the image of the zero section of $\mathfrak{M}$ ), is given by

$$
\gamma=\exp _{\nabla}[\operatorname{span}(v, J v) \cap W]
$$

at least in some neighborhood of the base point of $v$. We will often refer to "complex geodesics" merely as "geodesics", provided that the context is such that no confusion is likely to arise from this usage.

Let $(\mathfrak{M}, \mathfrak{g})$ be a complex-Riemannian manifold and $\nabla$ a holomorphic affine connection on $\mathfrak{M}$ satisfying $\nabla \mathrm{g}=0$ (but not necessarily $T_{\nabla}=0$ ). Then for any local holomorphic vector field $v$ satisfying $v\lrcorner \nabla v=0$, we have

$$
v\lrcorner \partial(g(v, v))=2 \mathfrak{g}(v, v\lrcorner \nabla v)=0,
$$

from whence it follows that a complex geodesic with a nonzero tangent $v$ at some point satisfying $\mathrm{g}(v, v)=0$ has the property that every tangent vector $w$ at any point of the geodesic satisfies $g(w, w)=0$. A holomorphic vector $v \in T^{\prime} \mathfrak{M}$ will be called null if $\mathfrak{g}(v, v)=0$, and a complex $\nabla$-geodesic with null tangents will be called a complex null $\nabla$-geodesic. If $T_{\nabla}=0$, we will simply call such a geodesic a complex null-geodesic.

3. Geodesics and symplectic geometry. In this section we will see a very different way of defining the geodesics of a complex-Riemannian manifold, using the ideas of Hamiltonian mechanics (cf. Arnold [1], Weinstein [16]). This alternate definition will prove useful as we concentrate our attention on the null geodesics.

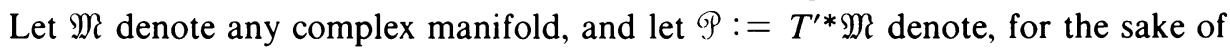
brevity, the total space of its holomorphic cotangent bundle. Then $P$ is equipped, by construction, with a holomorphic 1 -form $\theta \in \Gamma\left(\mathscr{P}, \Omega^{1}\right)$, called the canonical form of (P), defined by

$$
\left.\langle\theta, v\rangle\right|_{p}:=\left\langle p, \pi_{*} v\right\rangle \quad \forall p \in \mathscr{P},
$$

where $v$ is any local section of $T^{\prime} \mathscr{P}$ and $\pi: \mathscr{P} \rightarrow \mathfrak{M}$ is the canonical projection. If $\left(\tilde{z}^{1}, \ldots, \tilde{z}^{n}\right): \mathfrak{Q} \rightarrow \mathbf{C}^{n}$ is a holomorphic chart on $\mathfrak{M}$, then we may define holomorphic functions

$$
z^{j}:=\pi^{*} \tilde{z}^{j}, \quad \zeta_{j}: p \mapsto\left\langle p, \partial / \partial \tilde{z}^{j}\right\rangle, \quad j=1, \ldots, n,
$$

on $\pi^{-1}\left[\mathcal{U}\right.$ ], yielding a holomorphic chart $\left(z^{1}, \ldots, z^{n}, \zeta_{1}, \ldots, \zeta_{n}\right): \pi^{-1}[\mathcal{U}] \rightarrow \mathbf{C}^{2 n}$ on $\mathcal{P}$; with respect to these coordinates we have

$$
\left.\boldsymbol{\theta}\right|_{\pi^{-1}[\mathcal{Q}]}=\zeta_{j} \partial z^{j}
$$

It is now evident that the 2-form $\omega:=\partial \theta \in \Gamma\left(\mathcal{P}, \Omega^{2}\right)$, which will be called the (holomorphic) symplectic form of $\mathscr{P}$, induces an isomorphism $\lrcorner_{\omega}: T^{\prime} \mathscr{P} \stackrel{\cong}{\rightarrow} T^{*} \mathscr{P}$ : $v \mapsto \omega(v, \cdot)$ because, using the above local coordinates, $\left.\omega\right|_{\pi^{-1}[थ]}=\partial \zeta_{j} \wedge \partial z^{j}$.

Suppose now that $S \subset \mathcal{P}$ is a smooth complex hypersurface, i.e. a one-codimensional holomorphic submanifold of $\mathcal{P}$. Imitating the above procedure, we may define a map $\lrcorner_{\tilde{\omega}}: T^{\prime} S \rightarrow T^{\prime} S: v \mapsto \tilde{\omega}(v, \cdot)$, where $\tilde{\omega}$ denotes the restriction of $\omega$ to $S$. This time, however, we do not get an isomorphism; rather, the kernel $D \subset T^{\prime} S$ of $\lrcorner_{\tilde{\omega}}$ is a 
one-dimensional holomorphic distribution. (Since $\lrcorner_{\omega}$ is an isomorphism, $\operatorname{dim} D \leqslant$ $\operatorname{codim} S=1$; but $\operatorname{dim} D>0$ because $\tilde{\omega}$ is skew and $S$ is odd dimensional:

$$
\operatorname{det}\left[\tilde{\omega}_{j k}\right]=\operatorname{det}\left[-\tilde{\omega}_{k j}\right]=(-1)^{2 n-1} \operatorname{det}\left[\tilde{\omega}_{j k}\right]
$$

with respect to any local frame.) Therefore $D$ is tangent to a holomorphic foliation of $S$ by complex curves. These curves will be called the phase-space trajectories of $S$, and their images under $\pi: \mathscr{P} \rightarrow \mathfrak{M}$ will be called the trajectories of $S$.

Now suppose that $\mathfrak{M}$ is equipped with a complex-Riemannian metric $\mathfrak{g}$. Using the index-lowering map $b$ of $g$, we may define a smooth hypersurface

$$
\varepsilon_{t}:=b\left[\left\{v \in T^{\prime} \mathfrak{M} \mid v \neq 0, \mathfrak{g}(v, v)=t\right\}\right]
$$

for any $t \in \mathbf{C}$. The trajectories of $\mathcal{E}_{t}$ turn out to be surprisingly familiar objects.

Proposition. Suppose that $\gamma: \mathbf{U} \rightarrow \mathfrak{M}, U \subset{ }_{\text {open }} \mathbf{C}$, is a geodesic parameterized so that $\left.\gamma^{\prime}\right\lrcorner \nabla \gamma^{\prime}=0$, and let $t=\mathfrak{g}\left(\gamma^{\prime}, \gamma^{\prime}\right)$. Then the curve $\hat{\gamma}: \mathbf{U} \rightarrow \mathbb{P}: \zeta \rightarrow b \gamma^{\prime}(\zeta)$ is a phase-space trajectory of $\varepsilon_{t}$, and every space-space trajectory of any $\xi_{t}$ arises in this way. Thus, the geodesics (respectively, null geodesics) of $1 \mathrm{M}$ are just the trajectories of $\varepsilon_{0} \cup \varepsilon_{1}$ (respectively, $\varepsilon_{0}$ ).

Proof. We will check that $D$ of an arbitrary $\varepsilon_{1}$ is horizontal with respect to the torsion-free metric connection $\nabla$ and satisfies $\left.\pi_{*} D\right|_{b(p)}=[p]$ at a chosen (but arbitrary) $p \in \mathcal{E}_{t}$, thus implying the proposition. Choose "normal coordinates" $\left(z^{j}\right)$ about $\pi(p)$ so that $0 \in \mathbf{C}^{n}$ corresponds to $\pi(p)$ and affinely parameterized complex lines in $\mathbf{C}^{n}$ through 0 satisfy $\left.\gamma^{\prime}\right\lrcorner \nabla \gamma^{\prime}=0$. Then $\left.\left(\partial_{j} \mathrm{~g}^{k l}\right)\right|_{0}=0$ because $\Gamma_{j k}^{l}$ vanishes at 0 . But $\xi_{t} \cap \pi^{-1}\left[\mathcal{Q}_{1}\right]$ is given by $\left\{\left(\left(z^{j}\right),\left(\zeta_{j}\right)\right) \mid a^{j k} \zeta_{j} \zeta_{k}=t,\left(\zeta_{j}\right) \neq 0\right\}$, so that for $\left(z^{j}\right)=0$ the conormal bundle of $\xi_{t}$ is spanned by $\mathfrak{g}^{j k} \zeta_{j} d \zeta_{k}$. But since \lrcorner$_{\omega}\left(\mathfrak{a}^{j k} \zeta_{j} \partial / \partial z^{k}\right)=-\mathfrak{a}^{j k} \zeta_{j} d \zeta_{k}$, it follows that $\mathfrak{g}^{j k} \zeta_{j} \partial / \partial z^{k}$ spans $D$. Since the vanishing of $\Gamma_{j k}^{l}$ at 0 makes a vector $w$ at $\left((0),\left(\xi_{j}\right)\right)$ horizontal precisely if $\left\langle d \zeta_{j}, w\right\rangle=0 \forall_{j}, D$ is horizontal. And since $b\left(\left(\zeta_{j}\right)\right)=\mathfrak{g}^{j k} \zeta_{j} \partial / \partial \tilde{z}^{k}$, we are done.

\section{HOLOMORPHIC CONFORMAL GEOMETRY}

1. Conformal structures. Let $(\mathfrak{M}, \mathfrak{g})$ be a complex-Riemannian manifold. If $f$ : $\mathfrak{M} \underset{\mathrm{e}}{\rightarrow} \mathbf{C} \backslash\{0\}$ is a nonvanishing holomorphic function, $f \cdot g$ is also a holomorphic metric on $\mathfrak{M}$. We will say that two holomorphic metrics are conformally equivalent if one is obtained from the other in this fashion.

We will now consider a structure on a complex manifold which amounts locally to the specification of a conformal equivalence class of holomorphic metrics. We take the time to do so because our approach to complex-Riemannian geometry will entail viewing conformally invariant objects as more primitive than objects depending on the metric. ("Conformally invariant" just means "depending only on the conformal class".)

Let $\mathfrak{M}$ be a complex manifold. By a holomorphic conformal structure on $\mathfrak{M}$ we will mean a holomorphic line subbundle $\iota: E \hookrightarrow \odot^{2} T^{* * \mathfrak{M}}$ of the holomorphic quadratic forms on the tangent bundle such that the "index lowering" map b: $T^{\prime} \mathfrak{M} \rightarrow T^{* * \mathfrak{M}}$ $\left.\otimes E^{*}: v \mapsto v\right\lrcorner\left[\left(\iota \otimes \mathrm{id}_{E^{*}}\right)(\mathbf{1})\right]$ is an isomorphism, where $\lrcorner$ denotes contraction and 
where the complex number $\mathbf{1}$ is thought of as a section of the canonically trivial line bundle $E \otimes E^{*}$. A nonvanishing local section of $E$ will be a holomorphic metric on its domain of definition, and any two such sections are conformally equivalent on the intersection of their domains; such a section will be called a local representative metric of the conformal structure.

Keeping in mind that $E \otimes E^{*}$ is canonically trivial, we could instead define a conformal structure as a holomorphic metric taking values in a line bundle, namely $\left(\iota \otimes \mathrm{id}_{E^{*}}\right)(\mathbf{1}) \in \Gamma_{\mathfrak{R}} \vartheta\left(\left(\odot^{2} T^{* * \mathfrak{M})} \otimes E^{*}\right)\right.$. This is completely equivalent provided one introduces the obvious notion of the equivalence of two such forms as induced by isomorphism of the respective "value" line bundles.

EXAMPLE. To see a holomorphic conformal structure admitting no global representative metric, consider a complex vector space $\mathbf{V}$, of dimension at least 3 , equipped with a nondegenerate quadratic form $\mathbf{g} \in \odot^{2} \mathbf{V}^{*}$. Let $\mathbf{Q} \subset \mathbf{P V}$ be the quadric variety

$$
\mathbf{Q}=\{[v] \in \mathbf{P V} \mid \mathbf{g}(v, v)=0\} .
$$

Then $\mathbf{g}$ restricts to $\mathbf{Q}$ as a holomorphic metric of homogeneity 2. Moreover, this conformal structure is unique, for, by chern class considerations, the value linebundle of any conformal metric must be the square $\mathbf{H}^{\otimes 2}$ of the inverse Hopf bundle; but the restriction of such a metric to any projective line in $\mathbf{Q}$ is then a section of $\left(\Omega_{\mathbf{P}_{1}}^{1}\right)^{2}(2) \cong \mathcal{O}(-2)$ and so vanishes. But, by contrast, $\mathbf{Q}$ admits no global holomorphic metric because $c_{1}\left(T^{\prime} \mathbf{Q}\right) \neq 0$ (and $H^{2}(\mathbf{Q}, \mathbf{Z})$ is free).

The most intuitively clear picture of a holomorphic conformal structure is as a holomorphically varying assignment of "null cones" in the holomorphic tangent bundle. A holomorphic tangent vector $v \in T_{x}^{\prime} \mathfrak{M}$ on a complex manifold $\mathfrak{M}$ with conformal structure $E \subset \odot^{2}\left(T^{* * \mathfrak{M})}\right.$ is said to be null if $\mathfrak{g}_{x}(v, v)=0$ for some (and hence for any) nonzero element $g_{x}$ of $E_{x}$; in other words, a vector is null iff it is null for any local metric representing the conformal structure. The null cone at $x \in \mathfrak{M}$ is just the set of all null vectors in $T_{x}^{\prime} \mathfrak{M}$.

If we define the null quadric bundle of the conformal structure $E$ by

$$
\mathfrak{a}_{E}=\left\{[v] \in \mathbf{P} T^{\prime} \mathfrak{M} \mid v \text { null }\right\},
$$

where $\mathbf{P} T^{\prime} \mathfrak{M}$ is the projectivized holomorphic bundle and square brackets denote the projective equivalence class of a nonzero vector, then $\mathfrak{Q}_{E}$ is a holomorphic fiberbundle over $\mathfrak{M}$ with typical fiber the nonsingular quadric

$$
\mathbf{Q}_{n-2}=\left\{\left[z_{1}, \ldots, z_{n}\right] \in \mathbf{P}_{n-1} \mid \sum_{j=1}^{n} z_{j}^{2}=0\right\},
$$

where $n$ is the complex dimension of $\mathfrak{M}$. Elements of $\mathfrak{\beth}_{E}$ will be called null directions.

We now prove a converse to the above.

Proposition. Suppose that $\mathfrak{Q} \subset \mathbf{P} T^{\prime} \mathfrak{M}$ is a holomorphic bundle of nonsingular quadrics. Then $\mathfrak{D}$ is the null quadric bundle of a unique holomorphic conformal structure over $\mathfrak{M}$. 
Proof. Let $\Theta(2)$ be the sheaf on $\mathbf{P} T^{\prime} \mathfrak{M}$ of holomorphic functions of homogeneity 2 on the fibers. (In other words, local sections of $\Theta(2)$ are functions $f$ on conical regions of $T^{\prime} \mathfrak{M}$ which satisfy $f(\lambda v)=\lambda^{2} f(v)$ for $\lambda \in \mathbf{C}$.) Let $\theta_{\mathfrak{Q}}(2)$ be the sheaf of such functions restructed to $\mathfrak{\Omega}_{E}$, and let $\mathfrak{\Im}(2)$ be the ideal sheaf defined by $0 \rightarrow \mathfrak{J}(2) \rightarrow \vartheta(2) \rightarrow \vartheta_{\mathfrak{Q}}(2) \rightarrow 0$. What we must show is that any point $x \in \mathfrak{M}$ has a neighborhood $U$ such that there is a section $g \in \Gamma\left(\pi^{-1}(U)\right.$, $\left.\mathfrak{s}(2)\right)$ vanishing identically on no fiber of the canonical projection map $\mathbb{P} T^{\prime} \mathfrak{M} \rightarrow \mathfrak{M}$. For some $\mathfrak{g} \in \Gamma\left(U, \odot^{2} T^{*} \mathfrak{M}\right)$, we would then have $\mathbf{g}(v)=\mathfrak{g}(v, v)$, and $\mathfrak{g}$ would be a representative metric of the promised conformal structure.

Let us define sheaves $\mathfrak{V}$ and $\hat{\mathcal{V}}$ on $\mathfrak{M}$ as the following complete presheaves:

$$
\widetilde{V}_{U}=\Gamma\left(\pi^{-1}(U), \vartheta(2)\right), \quad \hat{\Upsilon}_{U}=\Gamma\left(\pi^{-1}(U), \vartheta_{\Omega}(2)\right) ;
$$

i.e. $\mathcal{V}=\pi_{*}^{0} \vartheta(2), \hat{\mathcal{V}}=\pi_{*}^{0} \mathcal{O}_{\mathcal{E}}(2)$ in the language of Godement [4]. I claim these sheaves are locally free and are in fact sections of vector bundles $V$ and $\hat{V}$ whose fibers are given by

$$
V_{x}=\Gamma\left(\pi^{-1}(x), \theta(2)\right), \quad \hat{V}_{x}=\Gamma\left(\pi^{-1}(x), \Theta_{\Omega}(2)\right) .
$$

In the case of $\mathcal{V}$ this follows immediately from the local triviality of $T^{\prime} \mathfrak{M}$. In the case of $\hat{\mathscr{V}}$ it is also immediate once we recognize that if $U_{1} \subset \mathfrak{M}$ is Stein and small enough so that $\left.\mathfrak{Q}\right|_{U_{1}}$ is trivial, we have

$$
H^{1}\left(\pi^{-1}\left(U_{1}\right) \cap \Omega, \theta\right)=H^{1}\left(\mathbf{Q}_{n-2} \times U_{1}, \theta\right)=0
$$

and so line bundles over $\left.\cong\right|_{U_{1}}$ are classified by their Chern classes; thus

$$
\Gamma\left(\pi^{-1}\left(U_{1}\right), \vartheta_{\mathfrak{Q}}(2)\right) \cong \Gamma\left(U_{1}\right) \otimes_{\mathbf{C}} \Gamma\left(\mathbf{Q}_{n-2}, \vartheta(2)\right) \text {. }
$$

If we now define $\subseteq$ to be the sheaf on $\mathfrak{M}$ defined by “pushing down" $\mathfrak{\Im}(2)$,

$$
\mathfrak{\subseteq}_{U}=\Gamma\left(\pi^{-1}(U), \mathfrak{s}(2)\right),
$$

we see that $\subseteq$ is kernel of a morphism $V \rightarrow \hat{V}$ of holomorphic vector bundles. But fiberwise, $V \rightarrow \hat{V}$ has 1-dimensional kernel as follows from the definitions. Hence $\subseteq$

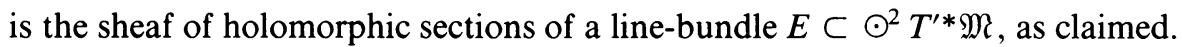

REMARK. The hypotheses of the proposition assumed that $\mathfrak{Q}$ was locally trivial. But Kodaira and Spencer [9] say that we need merely suppose that $\mathfrak{Q}$ is holomorphic and has fibers isomorphic to $\mathbf{Q}_{n-2}$, since the latter is a rigid manifold.

This then justifies our earlier statement that a holomorphic conformal structure is simply a holomorphic null-cone distribution.

\section{Null geodesics and conformal connections.}

Proposition. Let $(\mathfrak{M}, g)$ be a complex-Riemannian manifold and let $f: \mathfrak{M} \rightarrow \mathbf{C} \backslash\{0\}$ be a nonvanishing holomorphic function. Then $\mathrm{g}$ and $f \cdot g$ have the same null geodesics.

Proof. In I.3, it was shown that the trajectories of

$$
\mathcal{E}_{0}:=b\left[\left\{v \in T^{\prime} \mathfrak{M} \mid v \neq 0, \mathfrak{g}(v, v)=0\right\}\right]
$$

are precisely the null geodesics of $(\mathfrak{M}, \mathfrak{g})$. But replacing $g$ with $f \mathfrak{g}$ leaves $\mathcal{E}_{0}$ unchanged. 
Now if $(\mathfrak{M}, E)$ is a complex manifold with holomorphic conformal structure, we may construct a hypersurface $\mathcal{E}_{0} \subset T^{*} \mathfrak{M}$ by using the above prescription for all local sections of $E$. The null geodesics of $(\mathfrak{M}, E)$ are then defined to be the trajectories of $\mathcal{E}_{0}$. Thus, a curve is a null geodesic of $(\mathfrak{M}, E)$ iff it is inextendible and arises locally as a metric null geodesic of any local section of $E$.

EXAmple. Let $\mathbf{Q}_{n} \subset \mathbf{P}_{n+1}$ be a nonsingular quadric. Then the null geodesics of the canonical conformal structure on $\mathbf{Q}_{n}$ discussed in II.1 are just the projective lines in $\mathbf{P}_{n+1}$ contained in $\mathbf{Q}_{n}$.

REMARK. While the null geodesics themselves are conformally invariant, the "autoparallel" tangent fields $X$, satisfying $X\lrcorner \nabla X=0$, are not; indeed, the "real slices" of null geodesics, obtained by flowing along the real projections of the complex fields $X$, change if the metric is rescaled by a holomorphic function. However, the symplectic formulation makes it clear that the corresponding cotangent fields $\mathrm{g}(X, \cdot)$ are conformally invariant, since such a field, specified along a fixed null geodesic, is precisely a phase-space trajectory of $\mathcal{E}_{0}$. This fact will be pertinent in III.3.

Let $(\mathfrak{M}, E)$ be a complex manifold with holomorphic conformal structure, and let $\mathfrak{S}_{E} \rightarrow \mathfrak{M}$ be the null quadric bundle of $E$. If $\gamma$ is a null geodesic in $\mathfrak{M}$, and if we define the lift $\tilde{\gamma}$ of $\gamma$ to be

$$
\tilde{\gamma}=\left\{\left(x, T_{x}^{\prime} \gamma\right) \mid x \in \gamma\right\} \subset \mathfrak{\Omega}_{E},
$$

then the collection of all such lifts is a holomorphic foliation of $\mathfrak{Q}_{E}$ by complex curves.

More generally, a conformal connection $\tau$ on a complex manifold with holomorphic conformal structure $E$ will mean a family of null complex curves, precisely one of which is tangent to any null direction, such that the lifts foliate $\Omega_{E}$ holomorphically. Equivalently, a conformal connection is a holomorphic foliation of $\mathfrak{Q}_{E}$ by curves such that the tangent bundle $\mathfrak{D} \subset T^{\prime} \mathfrak{\beth}_{E}$ of the leaves satisfies $\pi_{* q} \mathfrak{D}=q$ $\forall q \in Q_{E}$, where $\pi: \mathfrak{Q}_{E} \rightarrow \mathfrak{M}$ is the canonical projection.

The curves of the family $\tau$ will be called $\tau$-geodesics.

EXAMPLE. Let $(\mathfrak{M}, \mathfrak{g})$ be a complex-Riemannian manifold, and let $\nabla$ be any holomorphic affine connection on $\mathfrak{M}$ such that $\nabla \mathfrak{g}=0$. Then the lifts of the null $\nabla$-geodesics foliate $\mathfrak{Q}$ holomorphically, and thus define a conformal connection.

Conversely, we have

THEOREM. Let $\tau$ be a conformal connection on $(\mathfrak{M}, E)$ and $\mathrm{g}$ a nonvanishing section of $E \subset \odot^{2} T^{* * \mathfrak{M}}$ on $U \subset \mathfrak{M}$. Then there exists a (nonunique and generally nonsymmetric) holomorphic affine connection $\hat{\nabla}$ on $U$ such that

(1) $\hat{\nabla} \mathrm{g}=0$, and

(2) the null $\hat{\nabla}$-geodesics are the (null) $\tau$-geodesics within $U$.

Proof. Let $\mathfrak{D}_{0}$ be the subbundle of $T^{\prime} \mathfrak{Q}_{E}$ tangent to the (standard) foliation of $\mathfrak{Q}_{E}$ by lifted null geodesics, and let $\mathfrak{D}$ denote the corresponding line distribution for $\tau$-geodesics. Since $\pi_{* q} \mathfrak{D}=\pi_{* q} \mathfrak{D}_{0}=q, \forall q \in \mathfrak{Q}_{E}, \mathfrak{D}-\mathfrak{D}_{0}$ is a holomorphic vector field $v$ of homogeneity 1 tangent to the fibers of $\pi$. 
Consider for a moment an $\theta(1)$ vector field $w$ on the standard quadric $\mathbf{Q} \subset \mathbf{P}_{n-1}$. (Recall that $\mathbf{Q}:=\left\{[z] \mid \sum_{z=1}^{n}\left(z^{j}\right)^{2}=0\right\}$.) Then $w$ is given by

$$
\left.w^{j}\right|_{[u]}=\Delta_{k l}^{j} u^{k} u^{l} \quad \forall u \in \mathbf{C}_{\cdot \ni}^{n} \cdot \sum_{j}\left(u^{j}\right)^{2}=0
$$

for a unique tensor $\Delta$ on $\mathbf{C}^{n}$ satisfying

$$
\Delta_{k l}^{j}=-\Delta_{j l}^{k}, \quad \Delta_{k j}^{j}=0, \text { and } \Delta_{k l}^{j}+\Delta_{l j}^{k}+\Delta_{j k}^{\prime}=0 .
$$

(Examination of the standard "Euler" exact sequence $0 \rightarrow \theta(1) \rightarrow \theta(2) \otimes \mathbf{C}^{n} \rightarrow$ $\theta\left(T^{\prime} \mathbf{P}_{n-1}\right) \times \theta(1) \rightarrow 0$ on $\mathbf{Q}$ yields such a representation-without algebraic constraints on $\Delta$-because $H^{1}(\mathbf{Q}, O(1))=0$; the result then follows from an easy, if tedious, tensor calculation.) By the same token, there is a unique holomorphic $\left(\begin{array}{l}1 \\ 2\end{array}\right)$-tensor field $\Delta$ on $U$ with

(a) $\mathfrak{g}_{j m} \Delta_{k l}^{m}=-\mathfrak{g}_{k m} \Delta_{j l}^{m}$,

(b) $\Delta_{k j}^{j}=0$,

(c) $g_{m\left[j \Delta_{k l}^{m}\right]}=0$

such that

$$
\left.v\right|_{u}=\Delta(u, u) \quad \forall u \cdot \ni \cdot[u] \in \mathfrak{\Omega}_{E} .
$$

Now define a connection $\hat{\nabla}$ on $U$ by

$$
X\lrcorner \hat{\nabla} Y:=X\lrcorner \nabla Y-\Delta(Y, X),
$$

where $\nabla$ is the Levi-Civita connection of $\mathrm{g}$. Then (a) implies $\hat{\nabla} \mathrm{g}=0$. We can therefore define the null geodesics of $\hat{\nabla}$ and the associated foliation of $\Omega_{E}$; call the corresponding direction field $\mathfrak{D}^{\prime}$. If $X$ is a vector field along a $\hat{\nabla}$ geodesic satisfying $X\lrcorner \hat{\nabla} X=0$, we have $X\lrcorner \nabla X=\Delta(X, X)$; so, if $X$ is null, the vertical part, with respect to $\nabla$, of the horizontal lift, with respect to $\hat{\nabla}$, of $X$ at the vector $X$ is just $\left.v\right|_{X}$. Hence $\mathfrak{D}^{\prime}=\mathfrak{D}$, proving the theorem.

REMARK. Notice that we have constructed a particular connection representing $\tau$, which is characterized by the conditions (a)-(c). Without such extra conditions, however, there is a certain freedom in the choice of $\hat{\nabla}$; for example, it is easy to verify that $\tilde{\nabla}$, defined by

$$
X\lrcorner \tilde{\nabla} Y=X\lrcorner \hat{\nabla} Y+A^{\#}(X, Y),
$$

where $A$ is a holomorphic 3-form and \# denotes the raising of an index with respect to $\mathrm{g}$, has the same geodesics as $\hat{\nabla}$ and satisfies $\tilde{\nabla}=0$.

Now notice that (a) and (c) imply that $\Delta_{l j}^{k}=2 \Delta_{[l k]}^{j}$ in an orthonormal frame. Hence $\langle Z, \Delta(X, Y)\rangle=\left\langle Y, T_{\hat{\nabla}}(Z, X)\right\rangle$, where $T_{\hat{\nabla}}$ is the torsion of $\hat{\nabla}$. (If we relaxed (c), the totally skew part of $\Delta$ would appear on the right with a factor of -2 .) We may therefore characterize the particular representative connection we have produced by the fact that $\hat{\Delta}=0$ and

(a) $\left(T_{\hat{\nabla}}\right)_{k j}^{j}=0$,

(b) $\left(T_{\hat{\nabla}}\right)_{\left[j k g_{\ell}\right] m}^{m}=0$.

Conversely, every holomorphic vector-valued 2-form satisfying (a) and (b) arises in this way for just one conformal connection. Also notice that if $g$ is replaced by $f \cdot g$, the field $T_{\hat{\nabla}}$ is unchanged; the argument is exactly that given for the proposition. 
We will now introduce a convenient abuse of language by identifying the conformal connection $\tau$ with the vector-valued 2-form $T_{\hat{\nabla}}$. In particular, we shall say that $\tau \equiv 0$ precisely if the $\tau$-geodesics coincide with the null geodesics.

3. Null geodesics as bicharacteristics. In this section we encounter yet another definition of a null geodesic. This definition, arising from the PDE concept of a bicharacteristic (cf., e.g., Guillemin and Sternberg [5]), has a venerable history going back to Monge. Our inclusion of it, however, is in fact the product of rather utilitarian motives-it will save us some work in III.3.

If $(\mathfrak{M}, E$ ) is a complex manifold with conformal structure, and if $\mathbf{S} \subset \mathfrak{M}$ is a nonsingular complex hypersurface, then we will say that $\mathbf{S}$ is characteristic or null iff every covector orthogonal to $\mathbf{S}$ is null; thus, $\mathbf{S}$ is null precisely if any local section of $E$ restricts to $\mathbf{S}$ as a degenerate quadratic form on each tangent space. A characteristic hypersurface $\mathbf{S}$ therefore comes equipped with a line subbundle $N \subset T^{\prime} \mathbf{S}$ of its tangent bundle which can be described either as the orthogonal space of $T^{\prime} \mathbf{S}$ (with respect to $E$ ) or as the kernel of the index-lowering map (associated with the degenerate induced conformal structure of $\mathbf{S}$ ). The integral curves of $N$ then give a canonical foliation of $\mathbf{S}$ by null curves, the so-called bicharacteristics of $\mathbf{S}$.

PROPOSITION. The bicharacteristics of any null hypersurface are null geodesics. Conversely, every null geodesic locally arises in this way.

Proof. As the assertion is local in character, we may assume that $\mathbf{S}$ is given by the vanishing of a holomorphic function $f$ with $d f \neq 0$, and also that the conformal structure is represented by a holomorphic metric g. If $\nabla$ denotes the standard connection associated with $g$, and if $v:=\operatorname{grad}(f)=(\nabla f)^{\#}$, then, because $S$ is null, one has $g(v, v)=f h$ for some holomorphic function $h$. Introducing indices as a bookkeeping device, with the convention that indices are to be raised and lowered with respect to $\mathfrak{g}$, we have

$$
\begin{aligned}
v^{a} \nabla_{a} v^{b} & \left.=v^{a} \nabla_{a} \nabla^{b} f=v^{a} \nabla^{b} \nabla_{a} f \quad \text { (because } T_{\nabla}=0\right) \\
& =v^{a} \nabla^{b} v_{a}=\frac{1}{2} \nabla^{b} v^{a} v_{a}=\frac{1}{2} \nabla^{b} f h=\frac{1}{2} h \nabla^{b} f+\frac{1}{2} f \nabla^{b} h \\
& =\frac{1}{2} h v^{b} \quad \text { when } f=0 .
\end{aligned}
$$

Thus the flow lines on $\mathbf{S}$ of the vector field $v$ are geodesic, as was to be proven.

Conversely, every null geodesic is locally contained in a null hypersurface to which it is orthogonal. To see this, let $U \subset \mathfrak{M}$ be geodesically convex with respect to some fixed metric $g$ representing the conformal structure on $U$ and letting $x \in U$ define $C_{x} \subset U$ to be the union of all null geodesics in $U$ through $x$; I then claim that $C_{x} \backslash\{x\}$ is a characteristic hypersurface having the null geodesics through $x$ as its bicharacteristics. It is obvious that $C_{x} \backslash\{x\}$ is a hypersurface because it is the (isomorphic) image, under the holomorphic exponential map of $\left\{v \in T_{x}^{\prime} \mathfrak{M} \mid v \neq\right.$ $0, \mathfrak{g}(v, v)=0\}$. By the use of this same exponential map and homogeneous coordinates on the null quadric of $x$ we can also find, for any fixed $u_{p} \in T_{p}^{\prime}\left(C_{x} \backslash\{x\}\right)$ and a fixed vector field $v$ on $C_{x}$ in a neighborhood of the null geodesic $\gamma$ connecting $x$ to $p$, with $v$ tangent to the null geodesics generating $C_{x}$ and satisfying $\nabla_{v} v=0$, a vector 
field $u$ defined in a neighbourhood of $\gamma$ with $u(p)=u_{p},[u, v]=0$, and $\lim _{y \rightarrow x} u(y)$ $=0$. Then

$$
\nabla_{v} \mathfrak{g}(u, v)=\mathfrak{g}\left(\nabla_{v} u, v\right)=\mathfrak{g}\left(\nabla_{u} v, v\right)=\frac{1}{2} \nabla_{u} \mathfrak{g}(v, v)=0
$$

and so $\mathfrak{g}(u, v) \equiv 0$. This shows that $C_{x}$ is characteristic and orthogonal to all its null geodesic generators, which proves the claim.

\section{SPACES OF COMPLEX NULl GEODESICS}

1. Civilized manifolds. Let $(\mathfrak{M}, \nabla)$ be a complex manifold with holomorphic connection. We will say that $\mathfrak{M}$ is geodesically convex with respect to $\nabla$ iff it is geodesically convex with respect to the underlying smooth connection of $\nabla$, i.e., iff each two points of $\mathfrak{M}$ are connected by a unique real geodesic of $\nabla$ varying continuously with the endpoints. By a classic theorem (Whitehead [17]), any manifold with smooth connection has a neighborhood basis consisting of geodesically convex sets; thus, the supposition of geodesic convexity imposes no local loss of generality on the geometry.

If $(\mathfrak{M}, E, \tau)$ is a complex manifold with holomorphic conformal connection, we define the space $\mathfrak{N}(\mathfrak{M}, E, \tau)$ of complex null geodesics of $(\mathfrak{M}, E, \tau)$ to be the set of leaves of the foliation of $\mathfrak{I}_{E}$ by lifted null $\tau$-geodesics with the quotient topology.

THEOREM. Let $(\mathfrak{M}, \mathfrak{g}, \nabla)$ be a complex-Riemannian manifold with metric connection for which it is geodesically convex. If $(E, \tau)$ is the conformal connection determined by $(\mathfrak{g}, \nabla)$, then $\mathfrak{R}(\mathfrak{M}, E, \tau)$ is a complex manifold in a unique way making the quotient map q: $\mathfrak{\beth}_{E} \rightarrow \mathfrak{N}$ a holomorphic map of maximal rank.

Proof. Let $\pi: \mathfrak{I}_{E} \rightarrow \mathfrak{M}$ be the canonical projection, $\xi \in \mathfrak{D}_{E}$ any point, $x:=\pi(\xi)$. We begin by constructing a neighborhood $W(\xi)$, which is the domain for a Frobenius chart for the foliation of $\mathfrak{D}_{E}$, by lifted $\tau$-geodesics and such that every fiber of $\mathbf{q}$ intersects $W$ in a path-connected set.

To this end, let $W_{0} \ni \xi$ be the domain of a chart

$$
\varphi: W_{0} \underset{e}{\stackrel{\Xi}{\rightarrow}} P \subset \mathbf{C}^{2 n-2}
$$

(where $P$ is a polydisc) such that $\varphi(\xi)=0$ and the components $\varphi^{2}, \ldots, \varphi^{2 n-2}$ are constant on every path-component of any fiber of $\mathbf{q}$ intersected with $W_{0}$; such complex Frobenius charts can easily be shown to exist by application of the smooth Frobenius theorem. Let $\mathbf{r}: \mathbf{C}^{2 n-2} \rightarrow \mathbf{C}^{2 n-3}$ be projection to the last $2 n-3$ components, and let $\mathbf{I}_{z}:=\varphi^{-1} \mathbf{r}^{-1}(z)$ for $z \in \mathbf{C}^{2 n-3}$, so that, in particular, $\mathbf{l}_{0}$ is the path-component in $W_{0}$ of the lifted null $\tau$-geodesics through $\xi$. Finally, let $U \subset \mathfrak{M}$ be a precompact $\nabla$-geodesically convex neighborhood of $x$ with smooth boundary $\partial U \approx S^{2 n-1}$, and let $U$ be chosen to be sufficiently small so that $U \cap \pi\left[\mathrm{l}_{0}\right]$ has frontier contained in $\partial U$.

Let $S:=W_{0} \cap \pi^{-1}[\partial U]$, and let $\hat{\mathbf{r}}:=\left.\mathbf{r} \varphi\right|_{S}: S \rightarrow \mathbf{C}^{2 n-3}$. Because $U \subset \mathfrak{M}$ is geodesically convex, $\hat{\mathbf{r}}^{-1}(z)$ is a connected smooth curve for all $z$ and, by construction, $\hat{\mathbf{r}}^{-1}(0) \approx S^{1}$. Since $\hat{\mathbf{r}}$ is a regular mapping, and because $\hat{\mathbf{r}}^{-1}(0)$ is compact, there is a tubular neighborhood $\mathbf{A}$ of $\hat{\mathbf{r}}^{-1}(0)$ such that $\mathbf{A} \approx \hat{\mathbf{r}}^{-1}(0) \times \mathbf{B}$ for $\mathbf{B} \subset \mathbf{C}^{2 n-3}$ in such a 
fashion that $\left.\hat{\mathbf{r}}\right|_{\mathbf{A}}$ looks like projection to $\mathbf{B}$; this is easily seen by covering $\hat{\mathbf{r}}^{-1}(0)$ with a finite number of Frobenius charts for the foliation of $S$ by the fibers of $\hat{r}$. Thus, by the Jordan curve theorem, for $z \in \mathbf{B}$ we have that $\hat{\mathbf{r}}^{-1}(z)$ then bounds a unique disc in $\mathbf{I}_{z}$, and $\pi$ carries this disc onto an entire null geodesic in $U$. Hence defining $W:=(\mathbf{r} \varphi)^{-1}(\mathbf{B}) \cap \pi^{-1}(U)$, we see that a path-component of any fiber of $\mathbf{q}$ in $W$ represents an entire null $\nabla$-geodesic in $U$, and so the geodesic convexity of $U$ guarantees that each fiber of $\mathbf{q}$ meets $W$ in a path-connected set. Hence the map $\mathbf{q r}^{-1}: \mathbf{B} \rightarrow \mathfrak{R}$ is an injection. By general properties of foliations, it is therefore a local homeomorphism, since $\mathbf{q}$ is an open map. Moreover, the transition functions between any two such local charts are holomorphic, as can be seen by using a finite sequence of overlapping Frobenius charts on $\mathfrak{\Omega}_{E}$ to compare any two such charts for $\mathfrak{R}$ with overlapping domains.

Thus, to prove the claim, all that really remains is to check that $\mathfrak{N}$ is a Hausdorff space. Because $\mathfrak{N}$ has a countable basis, it suffices to check that a sequence in $\mathfrak{N}$ can converge to at most one point. If we have a sequence of null geodesics $\gamma_{j}$ in $\mathfrak{M}$ converging to two distinct null geodesics $\gamma$ and $\hat{\gamma}$, we could then find $w_{j}, x_{j}, y_{j}$, $z_{j} \in \gamma_{j} ; w, x \in \gamma$; and $y, z \in \hat{\gamma}$ such that $w_{j} \rightarrow w, x_{j} \rightarrow x, y_{j} \rightarrow y$, and $z_{j} \rightarrow z$ in $\mathfrak{M}$. If $\mathscr{Q} \subset T \mathfrak{M}$ is the domain of the exponential map, and if $\hat{\mathscr{D}}=\operatorname{Re}^{-1}(\mathscr{D})$, where $\operatorname{Re}$ : $T^{\prime} \mathfrak{M} \cong T \mathfrak{M}: v \mapsto \frac{1}{2}(v+\bar{v})$, then $\varphi: \hat{\mathscr{D}} \rightarrow \mathfrak{M} \times \mathfrak{M}$ via $\varphi(v)=(\pi(v), \exp (\operatorname{Re}(v)))$ is a biholomorphism, where $\pi: T^{\prime} \mathfrak{M} \rightarrow \mathfrak{M}$ is the canonical projection. If we now look at the sequences $\varphi^{-1}\left(w_{j}, x_{j}\right)$ and $\varphi^{-1}\left(w_{j}, y_{j}\right)$, we notice that $\left[\varphi^{-1}\left(w_{j}, x_{j}\right)\right]=$ $\left[\varphi^{-1}\left(w_{j}, y_{j}\right)\right]$, where square brackets denote projective classes in $\mathbf{P} T^{\prime} \mathfrak{M}$. Since $\mathbf{P} T^{\prime} \mathfrak{M}$ is Hausdorff we therefore have

$$
\left[\varphi^{-1}(w, x)\right]=\lim _{j \rightarrow \infty}\left[\varphi^{-1}\left(w_{j}, x_{j}\right)\right]=\lim _{j \rightarrow \infty}\left[\varphi^{-1}\left(w_{j}, y_{j}\right)\right]=\left[\varphi^{-1}(w, y)\right],
$$

and so the null geodesic $\gamma=\exp \operatorname{Re}\left(\hat{\mathscr{D}} \cap \mathbf{C}^{-1}(w, x)\right)$ contains $y$. In the same way, $\gamma$ contains $z$. But since $\mathfrak{M}$ is geodesically convex, there is only one geodesic containing both $y$ and $z$, and that geodesic is, by hypothesis, $\hat{\gamma}$. Hence $\gamma=\hat{\gamma}$, and $\mathfrak{N}(\mathfrak{M}, E, \tau)$ is Hausdorff.

This concludes the proof.

From now on, we will say that $(\mathfrak{M}, E, \tau)$ is civilized if (a) $\mathfrak{N}(\mathfrak{M}, E, \tau)$ is a complex manifold, and if (b) $\mathbf{q}$ is injective on each fiber of the canonical projection $\mathfrak{S}_{E} \rightarrow \mathfrak{M}$. Evidently, geodesically convex neighborhoods are civilized.

2. The skies of $\mathfrak{M}$. Let $(\mathfrak{M}, E, \tau)$ be a complex $n$-manifold with civilized conformal connection. Associated with every point of $\mathfrak{M}$ is an $(n-2)$-quadric $\mathbf{Q}_{x} \subset \mathfrak{R}(\mathfrak{M}, E, \tau)$, given by $\mathbf{q}\left[\pi^{-1}(x)\right]$, which we will call the sky of $x$; thus, $\mathbf{Q}_{x}$ consists of all $\tau$-geodesics through $x$. (The term "sky", suggested by Yu. I. Manin, is inspired by Lorentzian geometry, where null geodesics represent light rays. The present author originally used the more cumbersome phrase "celestial quadric" [cf. LeBrun [10]] to refer to the same object.) If we choose to think of $\mathfrak{M}$ as $\left\{\mathbf{Q}_{x} \mid x \in \mathfrak{M}\right\}$, then the conformal connection $(E, \tau)$ is given to us for free, since $x_{j} \in \mathfrak{M}$ are points on a common $\tau$-geodesic iff $\bigcap_{j} \mathbf{Q}_{x_{j}} \neq \varnothing$. We shall presently see, however, that, if $n \neq 3,\left\{\mathbf{Q}_{x}\right\}$ is inherent to the complex manifold $\mathfrak{R}(\mathfrak{M}, E, \tau)$ : geometry on $\mathfrak{M}$ becomes global complex analysis on $\mathfrak{N}$. 
To make this precise, we begin with a

Proposition. Let $\mathbf{N} \rightarrow \mathbf{Q}_{x}$ be the normal bundle $\left(\left.T^{\prime} \Re\right|_{\mathbf{Q}_{x}}\right) / T^{\prime} \mathbf{Q}_{x}$. Then the holomorphic isomorphism type of $\mathbf{N}$ depends only upon the dimension $n$. Moreover, $\mathbf{N}$ satisfies:

(a)

$$
H^{0}(\vartheta(\mathbf{N})) \cong \begin{cases}\mathbf{C}^{n} & \text { if } n \neq 3 \text { and } n>1, \\ \mathbf{C}^{4} & \text { if } n=3,\end{cases}
$$

(b) $H^{1}(\Theta(\mathbf{N}))=0$,

(c) $H^{1}\left(\Theta\left(\mathbf{N} \otimes \mathbf{N}^{*}\right)\right)=0$.

Proof. We know that the jacobian of the quotient map q: $\mathfrak{\beth}_{E} \rightarrow \mathfrak{R}$ has maximal rank at all points, and $\mathbf{q}$ carries every fiber of $\pi: \mathfrak{Q}_{E} \rightarrow \mathfrak{M}$ isomorphically into $\mathfrak{R}$. The induced map from the normal bundle of $\pi^{-1}(x) \subset \mathfrak{Q}_{E}$ to that of $\mathbf{Q}_{x} \subset \Re$ is therefore surjective. But the normal bundle of $\pi^{-1}(x)$ is just the trivial bundle with fiber $T_{x}^{\prime} \mathfrak{M}$. Moreover, the horizontal lift of a null vector $v$ at $[v]$ is in the kernel of $\mathbf{q}_{*}$, which gives an inclusion of the universal line bundle $\mathbf{U} \rightarrow \mathbf{Q}_{x}$ into the kernel of $T_{x}^{\prime} \mathfrak{M} \rightarrow \mathbf{N}$. By a dimension count, we therefore have an exact sequence

$$
0 \rightarrow \mathbf{H}_{j}^{*} \underset{j}{\rightarrow} T_{x}^{\prime} \mathfrak{M} \rightarrow \mathbf{N} \rightarrow 0,
$$

where $\mathbf{H}$ is the restriction to $\mathbf{Q}_{x}$ of the hyperplane-section line-bundle on $\mathbf{P}\left(T_{x}^{\prime} \mathfrak{M}\right)$ and $j$ is the tautological inclusion. In particular,

$$
\left.\mathbf{N} \cong\left(T^{\prime} \mathbf{P}_{n-1}\right)\right|_{\mathbf{Q}_{n-2}} \otimes \mathbf{H}^{*} .
$$

The formulae (a)-(c) now follow by straightforward manipulation of the above exact sequence and the vanishing of $H^{p}\left(\vartheta\left(\mathbf{H}^{\otimes m}\right)\right)$ for $0<p<n-2$.

Corollary. If $n \neq 3$ the family $\left\{\mathbf{Q}_{x} \mid x \in \mathfrak{M}\right\}$ is complete in the sense of Kodaira [8].

REMARK. For $n=3$ the incompleteness of the family of skies has interesting consequences; cf. LeBrun [12].

Definition. Let $\mathfrak{X}$ be a complex $(2 m+1)$-manifold. A normal quadric in $\mathfrak{X}$ is an $m$-quadric embedded holomorphically in $\mathfrak{X}$ with normal bundle $\left.\left(T^{\prime} \mathbf{P}_{m+1}\right)\right|_{\mathbf{Q}_{m}} \otimes \mathbf{H}^{*}$.

Thus, $\mathbf{Q}_{x}$ is a normal quadric in $\mathfrak{R}(\mathfrak{M}, E, \tau)$.

THEOREM. Let $\mathfrak{X}$ be a complex $(2 m+1)$-manifold, $m \geqslant 2$, and let $\mathfrak{Y}$ be the set of normal quadrics in $\mathfrak{X}$. Then $\mathfrak{Y}$ is a complex $(m+2)$-manifold with conformal connection induced by intersection relations. If $\mathfrak{X}=\mathfrak{N}(\mathfrak{M}, E, \tau), \mathfrak{M} \subset \mathfrak{Y}$ as an open subset with the induced conformal connection.

Proof. That the space of all normal quadrics has the structure of a complex $(m+2)$-manifold (if $m \geqslant 2$ ) is a direct consequence of the proposition and Kodaira [8], for the latter tells one that the local moduli space of compact complex $m$-manifolds near a normal quadric has dimension $m+2$, using clauses (a) and (b) of the proposition; but (c) and the rigidity of quadrics imply, by Kodaira and Spencer [9], that a small deformation of a normal quadric is again a normal quadric. 
Now define a family of curves in the moduli space $\mathfrak{Y}$ by setting, for each $x \in \mathfrak{X}$, $\gamma_{x}:=\{\mathbf{Q} \in \mathfrak{V} \mid x \in \mathbf{Q}\}$. Indeed, if $3=\{(x, \mathbf{Q})|x \in \mathfrak{V}| x \in \mathbf{Q}\}$, then 3 is a holomorphic quadric bundle over $\mathfrak{Y}$, the "total space of the family $\mathfrak{Y}$ ", and the above curves are given by $\gamma_{x}=\operatorname{pr}_{2}\left(\mathrm{pr}_{1}^{-1}(x)\right)$, where $\mathrm{pr}_{1}$ and $\mathrm{pr}_{2}$ are the canonical projections of 3 to $\mathfrak{X}$ and $\mathfrak{Y}$, respectively. Moreover, again by general properties of complete analytic families, the jacobian of $\mathrm{pr}_{1}$ induces an isomorphism between $T_{\mathbf{Q}}^{\prime} \mathfrak{Y}$ and $H^{0}(\mathbf{Q}, \Theta(\mathbf{N}))$, where $\mathbf{N} \rightarrow \mathbf{Q}$ is the normal bundle in $\mathfrak{X}$. Because $\mathbf{N}$ is, by hypothesis, the standard normal bundle, there is a holomorphic conformal structure induced on $\mathfrak{Y}$ by defining $v \in T^{\prime} \mathfrak{Y}$ to be null iff the corresponding section of $\mathbf{N}$ has a zero. Further, this identifies 3 with $\mathfrak{\beth}_{E}$. The foliation of 3 by the fibers of $\mathrm{pr}_{1}$ then defines a conformal connection $\tau$ on $\mathfrak{Y}$ having the path components of the $\gamma_{x}$ 's as the $\tau$-geodesics.

Notice that we have not asserted that $\mathfrak{Y}$ is civilized; in fact, this need not be the case. If $\mathfrak{R} \subset \mathbf{C}^{4}$ is defined implicitly by

$$
\mathbf{C}^{4} \backslash \Re:=\left\{\left(z_{1}, z_{2}, z_{3}, z_{4}\right) \mid z_{1}+\bar{z}_{1}+i\left(z_{2}-\bar{z}_{2}\right)=z_{3}=z_{4}=0\right\},
$$

and if $\mathfrak{X} \subset \mathfrak{R}\left(\mathbf{C}^{4}, \Sigma d z_{j}^{2}, 0\right)$ is the set of all null lines meeting $\mathfrak{R}$, then $\mathfrak{Y}=\mathfrak{R}$. ( $\mathfrak{V} \subset \mathbf{C}^{4}$, as can be shown by noticing that $\mathfrak{R}\left(\mathbf{C}^{4}\right)$ is the complement of a normal 2-quadric in a hypersurface of bidegree $(1,1)$ in $\mathbf{P}_{3} \times \mathbf{P}_{3}$, and all normal quadrics in $\mathfrak{N}$ can thus be written explicitly as products of projective lines. But every point in the complement of $\Re$ is on a null line missing $\Re$, namely a null line of the form $z_{1}=-i z_{2}+\rho, z_{3}=z_{4}=0$, where $\rho$ is an arbitrary real constant.) But $\Re$ is not civilized, since its space of null geodesics is not Hausdorff-for the two null geodesics in $\Re$ described by

$$
\begin{aligned}
& z_{1}=i z_{2}, \quad z_{3}=z_{4}=0, \quad z_{1}+\bar{z}_{2}+i\left(z_{2}-\bar{z}_{2}\right)>0, \\
& z_{1}=i z_{2}, \quad z_{3}=z_{4}=0, \quad z_{1}+\bar{z}_{2}+i\left(z_{2}-\bar{z}_{2}\right)<0
\end{aligned}
$$

are both limit points of the sequence of null geodesics given by $z_{1}=i z_{2}+\zeta_{j}$, $z_{3}=z_{4}=0$ where $\zeta_{j} \in \mathbf{C}, \zeta_{j} \rightarrow 0$.

On the other hand, the situation is very far from disastrous; the failure of the Hausdorff property for the space of $\tau$-geodesics of $\mathfrak{Y}$ is all that can go wrong. If we say that $(\mathfrak{M}, E, \tau)$ is precivilized if $(\mathfrak{M}, E, \tau)$ is a possibly non-Hausdorff complex manifold, with conditions on $\mathbf{q}$ as before, then it is immediate that $\mathfrak{Y}$ is precivilized. This problem goes away completely, of course, if we allow, in contrast to our original definition, for some geodesics to be disconnected.

If $\mathfrak{M}$ is a connected complex $n$-manifold, $n>3$, with civilized conformal connection $(E, \tau)$, we shall define the dominion $\mathbf{D}(\mathfrak{M}, E, \tau)$ to be the space of all normal quadrics in $\mathfrak{R}(\mathfrak{M}, E, \tau)$, and the connected dominion $\mathbf{D}_{0}(\mathfrak{M}, E, \tau)$ to be the component of $\mathbf{D}(\mathfrak{M}, E, \tau)$ containing $\mathfrak{M}$.

A primary goal of the remaining part of this chapter will be to provide hypotheses under which $\mathbf{D}_{0}(\mathfrak{M}, E, \tau)=\mathfrak{M}$; it will be hoped that these hypotheses actually force $\mathfrak{M}=\mathbf{D}(\mathfrak{M}, E, \tau)$. Notice that some sort of hypotheses are necessary, since if $\mathfrak{M}$ is civilized and $x \in \mathfrak{M}, \mathfrak{M} \backslash x$ is also civilized and $\mathfrak{R}(\mathfrak{M})=\mathfrak{R}(\mathfrak{M} \backslash x)$, showing that $x \in \mathbf{D}(\mathfrak{M} \backslash x)$. 
Note, by the way, that the above definitions may be made if $n=2$, but the accompanying theory is not only trivial in character but also different from the high-dimensional case. (For example, $\mathbf{D}(\mathfrak{M})$ always has four components when $n=2$, while $\mathbf{D}(\mathfrak{M})$ is connected, at least for simple examples, when $n=4$.) As a matter of taste, we will ignore $n=2$.

3. Contact structures and torsion. In this section we characterize the vanishing of conformal torsion in terms of the existence of a contact form on $\mathfrak{R}$.

Let $\mathfrak{X}$ be a complex $(2 m+1)$-manifold. A holomorphic contact form on $\mathfrak{X}$ is a holomorphic 1 -form $\theta$ taking values in a line bundle $\mathbf{L} \rightarrow \mathfrak{X}$ and satisfying

$$
\theta \wedge(\partial \theta)^{\wedge m} \neq 0 \text {. }
$$

This expression may be interpreted simply as holding in terms of any local trivialization of $\mathbf{L}$; but since, for any holomorphic function $f$,

$$
f \theta \wedge(\partial(f \theta))^{\wedge m}=f \theta \wedge(f \partial \theta+\partial f \wedge \theta)^{\wedge m}=f^{m+1} \theta \wedge(\partial \theta)^{\wedge m},
$$

it follows that $\theta \wedge(\partial \theta)^{\wedge m}$ is a well-defined section of $\Omega^{(2 m+1)}\left(\mathbf{L}^{\otimes(m+1)}\right)$, and so, by $(* * *)$, one has

$$
\mathbf{L}^{\otimes(m+1)} \cong \mathrm{K}^{*},
$$

where $\mathrm{K} \rightarrow \mathfrak{X}$ is the canonical line bundle $\Lambda^{(2 m+1)} T^{\prime *} \mathfrak{X}$. In particular, the line bundle $\mathbf{L}$ is completely determined by $\mathfrak{X}$ if $H^{1}\left(\mathfrak{X}, \mathbf{Z}_{n+1}\right)=0$.

A holomorphic contact structure is an equivalence class of contact forms under the relation that $\theta \in \Gamma \Omega^{1}(\mathbf{L})$ is equivalent to $\hat{\theta} \in \Gamma \Omega^{1}(\hat{\mathbf{L}})$ iff there exists an isomorphism $\psi: \mathbf{L} \cong \hat{\mathbf{L}}$ such that $\psi \boldsymbol{\theta}=\hat{\boldsymbol{\theta}}$. More in the spirit of our description of conformal structures, one may instead say that a contact structure is a holomorphic distribution D of hyperplanes (i.e. the orthogonal spaces of some twisted holomorphic 1-form) for which the Frobenius integrability obstruction is nondegenerate. If $\mathfrak{X}$ admits such a structure, we will say that $\mathfrak{X}$ is of contact type.

REMARK. All holomorphic contact forms in dimension $2 m+1$ are locally equivalent to that determined by the form

$$
\alpha:=d z_{2 m+1}+\sum_{j=1}^{m} z_{j} d z_{j+m}
$$

on $\mathrm{C}^{2 m+1}$; a contact structure is a "flat" structure (cf., e.g., Arnold [1]). Thus, we may think of a contact structure on $X$ as a reduction of the maximal atlas of $X$ to a subatlas with transition functions $\Phi_{J K}$ satisfying $\Phi_{J K}^{*} \alpha=f \alpha$ for some holomorphic function $f$ in $\mathbf{C}^{2 m+1}$.

TheOREM. Let $\mathfrak{M}$ be of dimension $>3$. Then $\mathfrak{N}(\mathfrak{M}, E, \tau)$ is of contact type iff $\tau \equiv 0$. Moreover, the contact structure of $\mathfrak{R}(\mathfrak{M}, E)$ is unique.

Proof. Let us begin by showing that $\mathfrak{R}(\mathfrak{M}, E)$ is of contact type, which we will do by use of the notion of the reduction of a symplectic manifold (cf. Weinstein [16]). Using the notation of I.3, let $S \subset \mathscr{P}:=T^{*} \mathfrak{M}$ be a smooth complex hypersurface, $\omega \in H^{0}\left(\mathscr{P}, \Omega^{2}\right)$ the holomorphic symplectic form of $\mathcal{P}, \tilde{\omega}$ the restriction of $\omega$ to $S$, 
and $\left.D:=\operatorname{ker}(\lrcorner_{\tilde{\omega}}\right)$. We will suppose that the foliation of $S$ by phase-space trajectories tangent to $D$ has a complex manifold $\mathscr{F}$ as its space of leaves, and let $\rho: S \rightarrow \mathscr{F}$ be the quotient map. Then I claim that there is a 2 -form $\hat{\omega} \in H^{0}\left(\mathscr{F}, \Omega^{2}\right)$ such that $\rho^{*} \hat{\omega}=\tilde{\omega}$. Since $\tilde{\omega}$ is, by construction, normal to $D$, it suffices to show that if $v \in \mathcal{O}(D)$ then $L_{v} \tilde{\omega}=0$. But this is immediate from $d \tilde{\omega}=\left.d \omega\right|_{S}=0$, since $L_{v} \tilde{\omega}=$ $v\lrcorner d \tilde{\omega}+d(v\lrcorner \tilde{\omega})$. Thus $\tilde{\omega}=\rho^{*} \hat{\omega}$ for some 2 -form $\hat{\omega}$.

Since $\rho^{*} d \hat{\omega}=d \tilde{\omega}=0$, and since $\rho^{*}: \Omega_{\tilde{\sigma}}^{1} \rightarrow \Omega_{S}^{1}$ is an inclusion $\left(\rho_{*}\right.$ being a projection), $d \hat{\omega}=0$. Also, since $\left.\left.\left.\operatorname{rank}(\lrcorner_{\hat{\omega}}\right)=\operatorname{rank}(\lrcorner_{\tilde{\omega}}\right),\right\lrcorner_{\tilde{\omega}}: T^{\prime} \mathscr{F} \rightarrow T^{*} \mathscr{F}_{\mathcal{F}}$ is an isomorphism.

Now carry out the above construction for the hypersurface $\varepsilon_{0}$ of nonzero null covectors on $\mathfrak{M}$ when $\mathfrak{M}$ is equipped with a conformal structure $E$. Because the scalar multiplication map $m_{t}: T^{* * \mathfrak{M}} \rightarrow T^{*} \mathfrak{M}: \varphi \rightarrow t \varphi$, where $t \in \mathbf{C}_{*}:=\mathbf{C} \backslash\{0\}$, satisfies $m_{t}^{*} \omega=t \omega$, it will follow that the phase-space trajectories of $\mathcal{E}_{0}$ are taken onto phase-space trajectories by $m_{t}$. Indeed, the quotient $\mathscr{F} / \mathbf{C}_{*}$ is just $\mathfrak{N}(\mathfrak{M}, E)$, so that $\mathscr{F} \rightarrow \mathfrak{N}$ is a principal $\mathbf{C}_{*}$-bundle. Let us now define a line bundle $\mathbf{L} \rightarrow \mathfrak{N}$ by $\mathbf{L}:=\mathscr{F} \times \mathbf{C} / \mathbf{C}_{*}$, where $\mathbf{C}_{*}$ acts on $\mathscr{F} \times \mathbf{C}$ by the family of maps

$$
\hat{m}_{t}: \mathscr{F} \times \mathbf{C} \rightarrow \mathscr{F} \times \mathbf{C}:(x, s) \mapsto\left(m_{t} x, t s\right) ;
$$

thus, $\mathscr{F}$ is $\mathbf{L}^{*}$ with the zero-section removed. Since each fiber of the canonical projection $\lambda: \mathbf{L}^{*} \rightarrow \mathfrak{N}$ is a complex vector space, there is a standard holomorphic identification of $\lambda^{*}\left[\mathbf{L}^{*}\right]$ with the holomorphic tangent bundle of the fibers of $\lambda$, thus giving rise to a standard $\mathbf{C}_{*}$-invariant holomorphic vector field $X$ on $\mathscr{F}$ (i.e., on $\left.L^{*} \backslash 0_{\mathfrak{R}}\right)$, defined by $\left.X\right|_{p}:=\lambda^{*}(p)$. We may now define a 1 -form $\theta \in \Gamma \Omega^{1}(\mathbf{L})$ by $\left.\lambda^{*} \theta:=X\right\lrcorner \hat{\omega}$. This makes sense because $\left.X\right\lrcorner \hat{\omega}$ is normal to the fibers of $\lambda$ and satisfies

$$
\left.\left.\left.m_{t}^{*}(X\lrcorner \hat{\omega}\right)=X\right\lrcorner m_{t}^{*} \hat{\omega}=t(X\lrcorner \hat{\omega}\right),
$$

so that $\hat{\omega}(X, Y)$ is a section of $\mathbf{L}$ for any $\mathbf{C}_{*}$-invariant vector field $Y$.

Note that since $X$ may also be defined as the derivative of the complex 1-parameter group of biholomorphisms $t \mapsto m_{e^{t}}$, we also have

$$
L_{X} \hat{\omega}=d\left(e^{t} \hat{\omega}\right) /\left.d t\right|_{t=0}=\hat{\omega} .
$$

$\boldsymbol{\theta}$ is now a contact form on $\mathfrak{R}$. Indeed, we may locally trivialize $\mathbf{L} \rightarrow \mathfrak{R}$ by picking a local section of $\sigma$ of $\mathscr{F} \rightarrow \mathfrak{R}$, thus identifying $\theta$ with the $\mathrm{C}$-valued 1-form $\left.\sigma^{*}(X\lrcorner \hat{\omega}\right)$. But

$$
\begin{aligned}
\left.\left.\sigma^{*}(X\lrcorner \hat{\omega}\right) \wedge\left(d \sigma^{*}(X\lrcorner \hat{\omega}\right)\right)^{\wedge(n-2)} & \left.\left.=\sigma^{*}[(X\lrcorner \hat{\omega}) \wedge(d(X\lrcorner \hat{\omega})\right)^{\wedge(n-2)}\right] \\
= & \left.\left.\sigma^{*}[(X\lrcorner \hat{\omega}) \wedge\left(L_{X} \hat{\omega}\right)^{\wedge(n-2)}\right]=\sigma^{*}[X\lrcorner(\hat{\omega})^{\wedge(n-1)}\right]
\end{aligned}
$$

cannot vanish because $(\hat{\omega})^{\wedge(n-1)} \in \Gamma\left(\mathscr{F}, \Omega^{2 n-2}\right)$ is a nonvanishing holomorphic volume element (this being just an invariant way of saying $\operatorname{det}\left[\hat{\omega}_{j k}\right] \neq 0$ ), and $X$ is transverse to the image of $\sigma$.

Conversely, suppose that $\mathfrak{N}(\mathfrak{M}, E, \tau)$ admits a contact form $\theta: T^{\prime} \mathfrak{N} \rightarrow \mathbf{L}$. By a previous remark, $\mathbf{L}^{\otimes(n-1)}=\Lambda^{2 n-3} T^{\prime} \Re$. Thus

$$
\left.\mathbf{L}^{\otimes(n-1)}\right|_{\mathbf{Q}_{x}} \cong\left(\Lambda^{n-2} T^{\prime} \mathbf{Q}_{x}\right) \otimes\left(\Lambda^{n-1} \mathbf{N}\right)
$$


where $\mathbf{N} \rightarrow \mathbf{Q}_{x}$ is the normal bundle. But we saw in the previous section that $\left.\mathbf{N} \cong\left(\mathbf{H}^{*} \otimes T^{\prime} \mathbf{P}_{n-1}\right)\right|_{Q}$, where $\mathbf{Q}_{x}$ is identified with the standard quadric in $\mathbf{P}_{n-1}$ and $\mathbf{H} \rightarrow \mathbf{P}_{n-1}$ is the line bundle of chern class +1 . Hence

$$
\left.\mathbf{L}^{\otimes n-1}\right|_{\mathbf{Q}_{i}} \cong\left(\mathrm{K}_{\mathbf{Q}}^{*}\right) \otimes\left(\mathrm{K}_{\mathbf{Q}}^{*} \otimes \mathbf{H}_{\mathbf{Q}}^{-(n-2)}\right) \otimes\left(\mathbf{H}_{\mathbf{Q}}^{2} \times \mathbf{H}_{\mathbf{Q}}^{-1}\right) \cong \mathbf{H}_{\mathbf{Q}}^{n-1},
$$

where $\mathrm{K}_{\mathbf{Q}} \rightarrow \mathbf{Q}$ is the canonical bundle $\Lambda^{n-2} T^{*} \mathbf{Q}$ and $\mathbf{H}_{\mathbf{Q}} \rightarrow \mathbf{Q}$ is the restriction of $\mathbf{H} \rightarrow \overrightarrow{\boldsymbol{P}}_{n-1}$ to $\mathbf{Q}$. Hence $\left.\mathbf{L}\right|_{\mathbf{Q}_{x}}=\mathbf{H}_{\mathbf{Q}}$. However, $H^{0}\left(\mathbf{Q}_{m}, \Omega^{1}\left(\mathbf{H}_{\mathbf{Q}}\right)\right)=0$ provided that $m \geqslant 2$. (This is true because through every point of $\mathbf{Q}_{m}$ there passes an $(m-2)$ quadric's worth of projective lines contained in $\mathbf{Q}_{m}$, spanning the tangent space of $\mathbf{Q}_{m}$; and since $H^{1}\left(\mathbf{P}_{1}, \Omega^{1}(1)\right) \cong H^{1}\left(\mathbf{P}_{1}, \Theta(-1)\right)=0$, it follows that every global section of $\Omega_{\mathbf{Q}}^{1}\left(\mathbf{H}_{\mathbf{Q}}\right)$ is orthogonal to every such projective line and, so, vanishes.) Hence if $\mathfrak{R}(\mathfrak{M}, E, \tau)$ admits a holomorphic contact structure, every quadric $\mathbf{Q}_{x}$ is everywhere orthogonal to the contact form; in particular, if $\mathfrak{R}$ is of contact type, $\operatorname{span}\left\{T_{\gamma}^{\prime} \mathbf{Q}_{x} \subset T_{\gamma}^{\prime} \mathfrak{N} \mid x \in \gamma \subset \mathfrak{M}\right\}$ is not all of $T_{\gamma}^{\prime} \mathfrak{N}$. This will imply that $\tau \equiv 0$ by the argument that follows.

Suppose that $D \subseteq T^{\prime} \mathfrak{N}$ is a distribution of $(2 n-4)$-planes to which every $\mathbf{Q}_{x}$ is tangent in the sense that $T^{\prime} \mathbf{Q}_{x} \subset D$. Fix a particular $x_{1} \in \mathfrak{M}$ and define the light cone $C_{x_{1}}$ of $x_{1}$ to be, as in II.3, the set of points in $\mathfrak{M}$ connected to $x_{1}$ by $\tau$-geodesics

$$
C_{x_{1}}:=\left\{x_{2} \in \mathfrak{M} \mid \mathbf{Q}_{x_{1}} \cap \mathbf{Q}_{x_{2}} \neq \varnothing\right\} .
$$

Then $C_{x_{1}} \backslash x_{1}$ is a hypersurface in $\mathfrak{M}$ with tangent space at $x_{2}$ given by

$$
T_{x_{2}}^{\prime} C_{x_{1}}=\left\{\alpha \in H^{0}\left(\mathbf{Q}_{x_{1}}, \Theta(\mathbf{N})\right)|\alpha|_{\left.\mathbf{Q}_{x_{1}} \cap \mathbf{Q}_{v_{2}} \in D / T^{\prime} Q_{x_{2}}\right\}}\right.
$$

where $\mathbf{N}$ denotes, as before, the normal bundle $T^{\prime} \mathfrak{N} / T^{\prime} \mathbf{Q}_{x_{2}}$ of $\mathbf{Q}_{x_{2}}$, since $D / T^{\prime} \mathbf{Q}_{x_{2}}$ $=T^{\prime} \mathbf{Q}_{x_{1}}$ at $\mathbf{Q}_{x_{1}} \cap \mathbf{Q}_{x_{2}}$. But this expression depends only on the $\tau$-geodesic $\mathbf{Q}_{x_{1}} \cap$ $\mathbf{Q}_{x_{2}}$. Thus, if two points of $\mathfrak{M}$ are joined by a $\tau$-geodesic $\gamma$, their light cones are tangent along $\gamma$. Therefore $C_{x_{1}}$ is tangent to the null cone at $x_{2}$ (i.e. to the set of null vectors at $x_{2}$ ). The $\tau$-geodesics through $x_{1}$ are therefore contained in a hypersurface $C_{x}$ to which they are orthogonal, and so, by II.3, they are (torsion-free) null geodesics. So $\tau \equiv 0$.

4. Metric geometry: $*_{1}$-operators as cohomology classes. Let $(\mathfrak{M}, \mathfrak{g})$ be a complexRiemannian $n$-manifold. We will say that $e \in \Gamma \mathcal{O}\left(\Lambda^{n} T^{* * \mathfrak{M})}\right.$ is a holomorphic metric volume element for $(\mathfrak{M}, \mathfrak{g})$ if $\left(e\left(v_{1}, \ldots, v_{n}\right)\right)^{2}=1$ for one, and, hence, for every, orthonormal basis $\left\{v_{1}, \ldots, v_{n}\right\}$ for each holomorphic tangent space $T_{x}^{\prime} \mathfrak{M}, x \in \mathfrak{M}$. Clearly, if there is such a volume element, there are exactly two of them.

Notice that $(\mathfrak{M}, \mathfrak{g})$ will admit a holomorphic metric volume element if $H^{1}\left(\mathfrak{M}, \mathbf{Z}_{2}\right)$ $=0$, since $e$ is metric iff $e \otimes e=\alpha$, where, letting $\mathrm{K}:=\Lambda^{n} T^{*} \mathfrak{M}, \alpha \in \Gamma \odot\left(\mathrm{K}^{\otimes 2}\right)$ is defined by $\langle\alpha, \beta \otimes \beta\rangle:=n !\langle l(\beta), \beta\rangle$ for all $\beta \in \mathrm{K}^{*} ; l: \mathrm{K}^{*} \stackrel{\cong}{\rightarrow} \mathrm{K}$ is used in this expression to indicate the lowering of all indices with $\mathrm{g}$. (The canonical morphism $\mathrm{g} \rightarrow \alpha$, which is homogeneous of degree $n$ in $\mathrm{g}$ over $\theta$, also gives us an isomorphism $E^{\otimes n} \cong \mathrm{K}^{\otimes 2}$ on any complex $n$-manifold with conformal structure.)

Now let $(\mathfrak{M}, \mathfrak{g}, e)$ be a complex-Riemannian $n$-manifold with holomorphic metric volume element. The $*_{1}$-operator $*_{1}: T^{*} \mathfrak{M} \rightarrow \Lambda^{n-1} T^{*} \mathfrak{M}$ is defined by

$$
\left.{ }_{1}(\psi):=\psi^{\#}\right\lrcorner e,
$$


where $\#=(b)^{-1}$ is the index-raising map of $g$. We may observe that this operator determines $g$ up to an overall factor of $e^{2 \pi i k /(n-2)}, k \in \mathbf{Z}$ and, in particular, determines not only the conformal class of $\mathfrak{g}$, but also the Levi-Civita connection $\nabla$, provided that $n>2$. Indeed, in a local frame,

$$
e_{b \cdots c}^{a}\left(\lambda e^{d h \cdots c}\right)=\lambda(n-1) ! \mathfrak{g}^{a d},
$$

so the conformal class may be read off by contracting $*_{1}$ with an arbitrary element of $\mathrm{K}^{*}$; and since the conformal change $\mathrm{g} \rightarrow f \cdot \mathfrak{g}$ is accompanied by $*_{1} \rightarrow f^{(n-2) / 2} *_{1}$, the observation follows.

If $(\mathfrak{M}, E)$ is a complex $n$-manifold with conformal structure, $*: T^{*} \mathfrak{M} \rightarrow$ $\Lambda^{n-1} T^{*} \mathfrak{M}$ will be called a $*_{1}$-operator for $(\mathfrak{M}, E)$ if it locally arises as a $*_{1}$-operator in the former sense. If $n>2$ and $H^{1}\left(\mathfrak{M}, \mathbf{Z}_{n-2}\right)=0, a *_{1}$-operator always arises from a global metric and metric volume form. By a minor abuse of language, we will simply say that $*$ is in the conformal class $E$ if it is a $*{ }_{1}$ operator for $(\mathfrak{M}, E)$.

TheOREM. Let $(\mathfrak{M}, E)$ be a complex $n$-manifold, $n>3$, with civilized conformal structure for which every null geodesic is contractible. Let $\mathfrak{N}=\mathfrak{N}(\mathfrak{M}, E)$, and let $\mathbf{L} \rightarrow \mathfrak{N}$ be the value line-bundle of the contact form on $\Re$ as in $\S 3$. (Thus $\mathbf{L}^{* \otimes(n-1)}=\mathrm{K}$.) Then there is a natural one-one correspondence between (a) cohomology classes in $H^{n-2}\left(\mathfrak{N}, \Theta\left(\mathbf{L}^{* \otimes(n-2)}\right)\right)$ vanishing on no quadric $\mathbf{Q}_{x}$, and $(\mathrm{b}) *$, operators on $\mathfrak{M}$ in the conformal class $E$.

Proof. Let us begin by identifying $\Omega_{E}$ with the bundle $\Omega_{E}^{*}$ of null covectors by the index-lowering map $b$ of $E$, let $\hat{q}: \Omega_{E}^{*} \rightarrow \mathfrak{R}$ be given by $\hat{\mathbf{q}}=\mathbf{q} \circ(b)^{-1}$, and $\hat{\pi}$ : $\Sigma_{E}^{*} \rightarrow \mathfrak{M}$ by $\hat{\pi}=\pi \circ(b)^{-1}$, so that

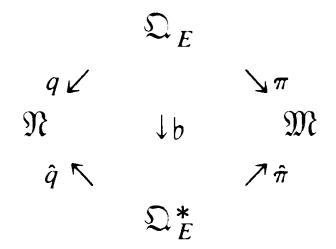

commutes. Then $\hat{\mathbf{q}}^{*} \mathscr{O}(\mathbf{L})=\mathscr{O}(1)$ in a natural way; cf. $\S 3$, proof of the theorem. Then, by duality,

$$
\begin{aligned}
H^{n-2}\left(\mathbf{Q}_{x}, \mathcal{O}\left(\mathbf{L}^{* \otimes(n-2)}\right)\right) & =H^{n-2}\left(\hat{\pi}^{-1}(x), \Theta(2-n)\right) \\
& =\left[H^{0}\left(\hat{\pi}^{-1}(x), \Omega^{n-2}(n-2)\right)\right] *
\end{aligned}
$$

But we may certainly produce a holomorphic volume element $\omega$ on $\hat{\pi}^{-1}(x)$ by setting, for all $v^{a}$ with $v^{a} v_{a}=0$,

$$
\left.\omega\right|_{v^{a}}:=w^{a} v_{b} e_{a}^{b c \cdots d} \forall w \cdot \ni \cdot w^{a} v_{a}=1,
$$

where $e_{a}^{b \cdots c}$ is in the fixed conformal class. Moreover, since this volume element never vanishes, we have given a holomorphic trivialization of $\left(\Lambda^{n-2} T^{* *} \mathbf{Q}_{n-2}\right) \otimes$ $\mathbf{H}^{\otimes n-2}$, so any other such volume element is perforce a constant multiple of the element we already have. Thus, since $e_{a}^{b \cdots c}$ is dual to $e_{b \cdots c}^{a}$, we have given a natural identification of $H^{n-2}\left(\mathbf{Q}_{x}, \Theta\left(\mathbf{L}^{*(n-2)}\right)\right) \backslash\{0\}$ with ${ }_{1}^{*}$-operators at $x$ in the conformal class $E$. We have defined a map $(\mathrm{a}) \rightarrow(\mathrm{b})$ by following through the above evaluation pointwise; we need to see now that this evaluation is an isomorphism. 
Let $\hat{\mathbf{q}}^{-1} \hat{C}\left(\mathbf{L}^{*(n-2)}\right)$ be the topological inverse sheaf of $\theta\left(\mathbf{L}^{*(n-2)}\right)$. There is a short exact sequence

$$
0 \rightarrow \hat{\mathbf{q}}^{-1} \mathcal{O}\left(\mathbf{L}^{*(n-2)}\right) \rightarrow \mathfrak{E}(2-n) \underset{d_{\hat{\mathbf{q}}}}{\rightarrow} \Omega_{\hat{\mathbf{q}}}^{1}(2-n) \rightarrow 0
$$

of sheaves on $\Omega_{E}^{*}$, where $d_{\hat{\mathbf{q}}}$ is differentiation up the fibers of $\hat{\mathbf{q}}$ and $\Omega_{\hat{\mathbf{q}}}^{1}$ is dual to holomorphic vector fields tangent to the fibers of $\hat{\mathbf{q}}$. But the index-raising map of $E$ identifies $\Omega_{\hat{\mathbf{q}}}^{1}$ with $\Theta(-1) \otimes \hat{\pi}^{*}(E)$. By the Leray spectral sequence of $\hat{\pi}$ (Godement $[4]), H^{p}\left(\Im_{E}^{*}, \Theta(-1) \otimes \hat{\pi}^{*}(\mathcal{C}(E))=0 \forall p\right.$, because $H^{p}\left(\mathbf{Q}_{n-2}, \vartheta(-1)\right)=0 \forall p$. Thus, the long exact sequence of the above short exact sequence is just the collection of isomorphisms

$$
H^{p}\left(\coprod_{E}^{*}, \hat{\mathbf{q}}^{-1} \odot\left(\mathbf{L}^{*(n-2)}\right)\right) \cong H^{p}\left(\coprod_{E}^{*}, \Theta(2-n)\right) .
$$

Again, by application of the Leray spectral sequence, our calculation of $H^{p}\left(\mathbf{Q}_{n-2}, \Theta(2-n)\right)$ for $p=n-2$ implies that

$$
H^{n-2}\left(\Im_{E}^{*}, \ominus(2-n)\right)=\Gamma \Omega^{n}\left(E^{*}\right) .
$$

Finally, the pull-back map

$$
\mathbf{q}^{-1}: H^{n-2}\left(\mathfrak{R}, \mathcal{O}\left(L^{*(n-2)}\right)\right) \rightarrow H^{n-2}\left(\mathfrak{\beth}_{E}^{*}, \mathbf{q}^{-1} \mathfrak{O}\left(\mathbf{L}^{*(n-2)}\right)\right)
$$

is an isomorphism (Buchdahl [3]) because the fibers of $\hat{\mathbf{q}}$ are contractible.

In fact, the above theorem holds even when $\tau \neq 0$, but the bundle $\mathbf{L} \rightarrow \varsigma \Re$ must then be defined in a rather unnatural way, using $\mathfrak{M}$. In fact, we may do this as follows: for every $\tau$-geodesic $\gamma \subset \mathfrak{M}$, let

$$
\left.\mathbf{L}_{\gamma}^{*}=\left\{\varphi \in \Gamma\left(\gamma, \Theta\left(\left.T^{*} \mathfrak{M}\right|_{\gamma}\right)\right) \mid \varphi^{\#} \text { is tangent to } \gamma \text { and } \varphi^{\#}\right\lrcorner \nabla \varphi \equiv 0\right\},
$$

where $\nabla$ is the canonical connection preserving some local metric $g$ and representing $\tau$. We must check that this is conformally invariant in order to see that it is well defined; but since a conformal change in $g$ induces exactly the same change in $\nabla$ as it does in the Levi-Civita connection, the necessary calculation has already been performed in II.2. Having defined $\mathbf{L}$, it is immediate that $\hat{\mathbf{q}}^{*} \Theta(\mathbf{L})=\Theta(1)$, which is the only property required for the proof of the theorem.

5. Reflexive manifolds. Recall that we defined the dominion $\mathbf{D}$ and connected dominion $\mathbf{D}_{0}$ of a manifold with civilized conformal connection in $\S 2$. We now define a complex manifold $\mathfrak{M}$ with civilized conformal connection $(E, \tau)$ to be reflexive (respectively, c-reflexive) if $\mathfrak{M}=\mathbf{D}(\mathfrak{M}, E, \tau)$ (respectively, $\mathfrak{M}=\mathbf{D}_{0}(\mathfrak{M}, E, \tau)$ ).

THEOREM. Let $\mathfrak{M}$ be a complex $n$-manifold, $n>3$, with civilized conformal connection $(E, \tau)$ and satisfying:

(1) $\mathfrak{M}$ is Stein;

(2) $\mathrm{K} \otimes E^{*}$ is trivial;

(e) every null geodesic is contractible.

Then $(\mathfrak{M}, E, \tau)$ is c-reflexive.

Proof. Because of condition (3), every holomorphic section of $\mathrm{K} \otimes E$ is obtained (cf. §4) by integrating an element of $H^{n-2}\left(\mathfrak{R}, \Theta\left(\mathbf{L}^{*(n-2)}\right)\right)$ over all skies. But such integration may be carried out over all normal quadrics, yielding a holomorphic 
section of some line-bundle $\mathbf{V} \rightarrow \mathbf{D}(\mathfrak{M}, E, \tau)$ with $\left.\mathbf{V}\right|_{\mathfrak{M}}=E^{*} \otimes \mathrm{K}$; thus, the restriction map

$$
\Gamma\left(\mathbf{D}_{0}(\mathfrak{M}, E, \tau), \Theta(\mathbf{V})\right) \rightarrow \Gamma\left(\mathfrak{M}, \Theta\left(\mathrm{K} \otimes E^{*}\right)\right)
$$

is an isomorphism. Let ${ }^{*}$, be a global nonvanishing section $\theta(\mathrm{K} \otimes E)$ on $\mathfrak{M}$, and let $\sigma$ be its extension to $\mathbf{D}_{0}(\mathfrak{M}, E, \tau)$. Let $\mathbf{W} \subset \mathbf{D}_{0}(\mathfrak{M}, E, \tau)$ denote the zero locus of $\sigma$, and let $\partial \mathfrak{M}$ denote the frontier of $\mathfrak{M}$ in $\mathbf{D}_{0}(\mathfrak{M}, E, \tau)$.

I claim that $\partial \mathfrak{M} \subset \mathbf{W}$. For if $x \in \partial \mathfrak{M}$ with $\sigma(x) \neq 0$, let $x_{j} \in \mathfrak{M}$ be a sequence with $x_{j} \rightarrow x$, and let $f$ be a holomorphic function on $\mathfrak{M}$ with $f\left(x_{j}\right) \rightarrow \infty$, using condition (1). Letting $\sigma_{1}$ denote the extension of $f *_{1}$ to $\mathbf{D}_{0}(\mathfrak{M}, E, \tau)$,

$$
\sigma(x)=\lim _{j \rightarrow \infty} *_{1}\left(x_{j}\right)=\left(\lim _{j \rightarrow \infty} f^{-1}\left(x_{j}\right)\right)\left(\lim _{j \rightarrow \infty} \sigma_{1}\left(x_{j}\right)\right)=0 \sigma_{1}\left(x_{j}\right)=0 .
$$

By the same argument, the extension of any holomorphic section of $\mathrm{K} \otimes E$ to $\mathbf{D}_{0}(\mathfrak{M})$ must vanish identically on $\partial \mathfrak{M}$.

But now, since $\partial \mathfrak{M} \subset \mathbf{W}$, it has real codimension at least 2 , so $\mathbf{D}_{0}(\mathfrak{M})-\partial \mathfrak{M}$ is connected. Therefore $\mathbf{D}_{0}(\mathfrak{M})=\mathfrak{M} \cup \partial \mathfrak{M}$, and since by construction $\mathbf{W} \cap \mathfrak{M}=\varnothing$, $\partial \mathfrak{M}=\mathbf{W}$. Let $x \in \mathbf{W}$ be a nonsingular point, and let $z$ be a function on a neighborhood of $x$ with $d z \neq 0$ such that $z=0$ defines $\mathbf{W}$ near $x$. If $x_{j}$ is a sequence of points of $\mathfrak{M}$ converging to $x$, and if $f$ is a holomorphic function on $\mathfrak{M}$ tending to infinity along $\left\langle x_{j}\right\rangle$, let $\sigma_{m}$ be the extension of $f^{m} *_{1}$ to $\mathbf{D}_{0}(\mathfrak{M})$ for $m$ positive integers. In a neighborhood of $x$, for some nonnegative integers $k_{m}$ and some nonzero sections $g_{m}$ of $\mathbf{V}$, we have $\sigma_{m}=z^{k_{m}} g_{m}$. But $f=\sigma_{m} / \sigma_{m-1}=z^{k_{m}-k_{m-1}}\left(g_{m} / g_{m-1}\right)$ is singular when $z=0$, so $k_{m}<k_{m-1} \forall m$, which is a contradiction since all the $k_{m}$ must be nonnegative. Thus $\mathbf{W}$ can have no smooth points and so is empty.

ADDED ACKNOWLEDGEMENT. I would like to thank T. Ekedahl for pointing out a flaw in the original version of the proof.

REMARKs. (a) Notice that hypothesis (2) is only topological in nature, since $\mathfrak{M}$ is assumed to be Stein; one just needs $c_{1}\left(\mathrm{~K} \otimes E^{*}\right)=0$. If $\mathfrak{M}$ is simply connected, this happens precisely if $c_{1}(\mathfrak{M})=0$.

(b) If $\tau=0, \mathrm{~V}=\left(\mathrm{K} \otimes E^{*}\right)_{D}$.

(c) If $\mathfrak{M}$ is geodesically convex with respect to some metric representing $(E, \tau)$, then $\mathfrak{M}$ will automatically satisfy the hypotheses of the theorem; in fact, (2) and (3) are trivial. As for showing that $\mathfrak{M}$ is Stein, this follows from the observation that an exhaustion of $\mathfrak{M}$ by geodesically convex regions is an exhaustion by holomorphically convex regions.

(To see that a precompact geodesically convex region $\mathfrak{R}$ is holomorphically convex, consider a complex hypersurface $\mathfrak{X}$ in $\mathfrak{M}$ obtained by exponentiating a hyperplane in the tangent space of some point $\xi$ on the boundary of $\Re ; \mathfrak{X}$ can always be chosen so that $\mathfrak{X} \cap \mathfrak{R}=\varnothing$, using the fact that $\mathfrak{R}$ is geodesically convex. $\mathfrak{X}$ is now defined by a global holomorphic function $z$, and $\left.z^{-1}\right|_{\Re}$ is a holomorphic function on $\Re$ blowing up at $\xi$.)

CONJECTURE. If ( $\mathfrak{M}, E, \tau)$ is $c$-reflexive, it is reflexive. 
6. Deformation theory. In this section, we consider deformations of the space $\mathfrak{R}$ of complex null geodesics of a civilized manifold, showing that, suitably interpreted, a small deformation of a space of complex null geodesics is again a space of complex null geodesics.

Recall that $(\mathfrak{M}, E, \tau)$ was defined to be precivilized if $\mathfrak{R}(\mathfrak{M}, E, \tau)$ is a possibly non-Hausdorff complex manifold. In this case, we will say that a complex manifold $\hat{\mathfrak{R}}$ is a generalized space of $\tau$-geodesics for $(\mathfrak{M}, E, \tau)$ if there is a surjective local biholomorphism $\mathfrak{R} \rightarrow \hat{\mathfrak{N}}$ which is injective on every sky $\mathbf{Q}_{x}$. Thus, points of $\hat{\mathfrak{R}}$ are collection of disjoint $\tau$-geodesics.

THEOREM. Let $\mathbf{p}: \mathfrak{R} \rightarrow \mathbf{C}$ be a holomorphic map of maximal rank such that $\mathbf{p}^{-1}(0) \cong \mathfrak{N}(\mathfrak{M}, E, \tau)$ for some civilized complex $n$-manifold $\mathfrak{M}, n \neq 3$, with civilized conformal connection $(E, \tau)$. Then there is a neighborhood $\tilde{\mathfrak{X}}$ of $\mathbf{p}^{-1}(0)$ in $\Re$ such that $\mathbf{p}^{-1}(t) \cap \tilde{\mathscr{T}}$ is a generalized space of $\tau_{t}$-geodesics for some complex $n$-manifold $\mathfrak{M}_{t}$ with conformal connection $\left(E_{t}, \tau_{t}\right)$.

Proof. This is very much like the theorem of III.2. We will define a quadric $\mathbf{Q} \subset \mathcal{N}$ to be $\mathbf{p}$-normal if the normal bundle of $Q$ relative to the fibers of $\mathbf{p}$ is the standard bundle $\mathbf{N}$ of III.2. (This makes sense because the function $\left.\mathbf{p}\right|_{\mathbf{Q}}$ is necessarily constant by the maximum principle.) Because $H^{1}(\mathbf{Q}, \mathbf{N} \oplus \mathbf{C})=0$ and $\operatorname{dim}_{\mathbf{C}} H^{0}(\mathbf{Q}, \mathbf{N} \oplus \mathbf{C})=n+1$, the rigidity of $\mathbf{Q}$ and $\mathbf{N}$ implies that the parameter space for all $p$-normal quadrics is a complex $(n+1)$-manifold $\Re$. Moreover, the induced projection $\hat{\mathbf{p}}$ : $\mathfrak{\Re} \rightarrow \mathbf{C}$ is of maximal rank. Let $\tilde{\mathscr{T}}$ be the set swept out by quadrics in the family $\Re$. Then $\tilde{\mathscr{T}}$ is open, because every point in $\mathbf{N} \oplus \mathbf{C}$ is the value of some local section. By Theorem III.2, the result follows.

7. Reality structures, $C R$-manifolds, and all that. Finally, we consider the extra bit of structure that arises on the space of null geodesics of a civilized complexification. If $\mathfrak{S}: \mathfrak{M} \rightarrow \overrightarrow{\mathfrak{C}} \rightarrow \mathfrak{M}$ is a complex conjugation $\left(\mathfrak{C}^{2}=\mathbf{1}\right)$ carrying some conformal structure $E$ onto its complex conjugate, then $\mathbb{5}$ carries null geodesics to null geodesics (because null hypersurfaces are taken to null hypersurfaces, and the kernel of the restricted index-lowering map on such a surface will be carried onto the complex conjugate of the corresponding kernel by (5), so for $\mathfrak{M}$ civilized there is an induced map $\hat{\mathfrak{C}}: \mathfrak{N}(\mathfrak{M}, E) \underset{\bar{\theta}}{\rightarrow} \mathfrak{N}(\mathfrak{M}, E)$ satisfying $\hat{\mathfrak{C}}^{2}=1$; we will call such a map a reality structure for $\mathfrak{R}$. The "real" skies $\mathbf{Q}_{x}$ satisfying $\hat{\mathfrak{C}}\left[\mathbf{Q}_{x}\right]=\mathbf{Q}_{x}$ correspond precisely to the real points of $\mathfrak{M}$. Fixed points of $\hat{\mathfrak{C}}$ correspond to real null geodesics of the induced pseudo-Riemannian conformal structure on the real slice $\mathbf{M} \subset \mathfrak{M}$; thus, if $\mathbf{M}$ is Riemannian, $\hat{\mathfrak{C}}$ acts without fixed points.

This picture carries through to the more general case when $\mathbf{M}$ is endowed with a real conformal torsion tensor $\tau_{\mathbf{R}}$, which can then be extended to some complexification as a holomorphic conformal torsion tensor $\tau$; it is more-or-less immediate that the $\tau$-geodesics are carried onto $\tau$-geodesics. The distinguishing feature of the torsion-free case is, as pointed out in $\$ 3$, the presence of a holomorphic contact structure on $\mathfrak{M}$; this structure is automatically taken onto its complex conjugate by $\hat{\mathfrak{s}}$. 
Conversely, if $\mathfrak{X}$ is a complex $(2 m+1)$-manifold, $m \geqslant 2$, acted on by a antiholomorphic involution $\hat{\mathfrak{F}}$, there is an induced antiholomorphic involution on the space $\mathfrak{Y}$ of all normal quadrics in $\mathfrak{X}$, giving $\mathfrak{Y}$ the structure of a complexification of the set of fixed quadrics, which then inherits a pseudo-Riemannian conformal structure from $\mathfrak{Y}$.

To see that this discussion is not devoid of content, consider a real-analytic pseudo-Riemannian manifold $(\mathbf{M}, \mathbf{g})$ with complexification $(\mathfrak{M}, \mathfrak{g})$, and let $U \subset \mathfrak{M}$ be a geodesically convex subregion meeting $\mathbf{M}$. Then $\hat{U}:=U \cap \mathfrak{F}[U]$ is also geodesically convex and nonempty. $(\hat{U},[\mathrm{~g}])$ then has an induced reality structure. Moreover, by $\S 5$ we know that $U \cap M$ is at least an entire connected component of the set of real normal quadrics in $\Re(\hat{U},[g])$.

If we suppose that $(\mathbf{M}, \mathbf{g})$ is Riemannian, then the union of all real skies in $\mathfrak{R}(\hat{U},[\mathrm{~g}])$ is a real-analytic $C R$-manifold (of real dimension $3 n-4$ and of generic type) which can be identified (as a smooth manifold) with the quadric bundle of null directions in $\left.\mathbf{P}\left(\mathbf{C} \otimes_{\mathbf{R}} T \mathbf{M}\right)\right|_{\mathbf{M} \cap U}$. In fact, the $C R$ distribution is spanned by the holomorphic tangent spaces of the fibers (skies) and the 1-dimensional distribution given at $[v]$ by the horizontal lift of $[\bar{v}]$. Thus, we may glue all of these locally defined $C R$-manifolds together to associate a single real-analytic $C R$-manifold to all of $\mathbf{M}$. Embedding this manifold creates a precivilized complexification for $\mathbf{M}-$ the space of all normal quadrics in this ambient space.

As one might expect, this $C R$-manifold can be constructed even when $\mathbf{M}$ is merely smooth, but now the $C R$-manifold is not embeddable unless the original conformal structure is real-analytic with respect to some atlas. But this is really another story altogether, and it will be more appropriate to give a separate account of it elsewhere.

Appendix. Metrics as cohomology classes. In light of III.4, it seems reasonable to ask if metrics - as opposed to the closely related $*_{1}$-operators-can be represented as cohomology classes on the space of null geodesic $\mathfrak{R}$. In fact, this can be done.

TheOREM. Let $(\mathfrak{M}, E)$ and $\mathbf{L} \rightarrow \mathfrak{N}(\mathfrak{M}, E)$ be as in III.4. Then

$$
H^{1}\left(\mathfrak{R}, \Theta\left(\mathbf{L}^{*}\right)\right)=\Gamma(\mathfrak{M}, \vartheta(E)) .
$$

Thus, metrics in the conformal class $E$ correspond to elements of $H^{1}\left(\Re, O\left(L^{*}\right)\right)$ vanishing on no sky $Q x, x \in \mathfrak{M}$.

Proof. The short exact sequence on $\mathfrak{\beth}^{*}$,

$$
0 \rightarrow \hat{\mathbf{q}}^{-1} \mathcal{O}\left(\mathbf{L}^{*}\right) \rightarrow \mathcal{O}(-1) \underset{d_{\hat{\mathbf{q}}}}{\rightarrow} \Omega_{\hat{\mathbf{q}}}^{1}(-1) \rightarrow 0,
$$

yields the isomorphism $\delta: H^{0}\left(\Omega_{\hat{\mathbf{q}}}^{1}(-1)\right) \stackrel{\cong}{\rightarrow} H^{1}\left(\hat{\mathbf{q}}^{-1} \mathcal{O}\left(\mathbf{L}^{*}\right)\right)$ because $H^{0}(\mathcal{O}(-1))=$ $H^{1}(\vartheta(-1))=0$. Since $\Omega_{\hat{q}}^{1}(-1)=\pi^{*} \vartheta(E)$, the theorem follows.

Note that the isomorphism cannot be realized by integrating over skies. Thus, it does not appear that the present theorem could easily be employed to produce an alternative version of III.5. 


\section{REFERENCES}

I. V. I. Arnold, Mathematical methods of classical mechanics, Springer-Verlag, Berlin, 1978. (Russian: "Nauka", Moscow, 1974)

2. M. Atiyah, N. Hitchin and I. M. Singer, Self-duality in four-dimensional Riemannian geometry, Proc. Roy. Soc. London Ser. A 362 (1978), 425-461.

3. N. P. Buchdahl, On the relative de Rham sequence, Proc. Amer. Math. Soc. 87 (1983), 363-366.

4. R. Godement, Topologie algébrique et théorie des faisceaux, Hermann, Paris, 1958.

5. V. Guillemin and S. Sternberg, Geometric asymptotics, Math Surveys, No. 14, Amer. Math. Soc., Providence, R. I., 1977.

6. N. Hicks, Notes on differential geometry, Van Nostrand-Reinhold, Princeton, N. J., 1971.

7. J. Isenberg. P. Yasskin and P. S. Green, Non-self-dual gauge fields, Phys. Lett. B 78 (1978), $462-464$.

8. K. Kodaira, $A$ theorem of completeness of characteristic systems for analytic families of compact submanifolds of complex manifolds, Ann. of Math. (2) 75 (1962), 146-162.

9. K. Kodaira and D. C. Spencer, On deformations of complex analytic structures. I, II, Ann. of Math. (2) 67 (1958), 328-466.

10. C. R. LeBrun, Spaces of complex geodesics and related structures, Ph.D. Thesis, Oxford, 1980.

11. (1982). 345-354. The first formal neighborhood of ambitwistor space for curved space-time, Lett. Math. Phys. 6

12. H-space with a cosmological constant, Proc. Roy. Soc. London Ser. A 380 (1982), 171-185.

13. Yu. I. Manin, Sovremennye problemy matematiki, Vol. 17, "Nauka”, Moscow, 1981.

14. J. Milnor and J. Stasheff, Characteristic classes, Ann. of Math. Studies, No. 76, Princeton Univ. Press, Princeton, N.J., 1974.

15. R. Penrose, Nonlinear gravitons and curved twistor theory, Gen. Relativity and Gravitation 7 (1976), $31-52$.

16. A. Weinstein, Lectures on symplectic manifolds, CBMS Regional Conf. Ser. in Math., no. 29, Amer. Math. Soc., Providence, R. I., 1977.

17. J. H. C. Whitehead, Convex regions in the geometry of paths, Quart. J. Math. 3 (1932), 33-42.

18. E. Witten, Phys. Lett. B 77 (1978), 394-397.

Mathematical Sciences Research Institute, University of California, Berkeley, California 94720 